\title{
. \\ CAPM CONDICIONAL COM APRENDIZAGEM APLICADO AO MERCADO BRASILEIRO DE AÇÕES
}

\section{JOÃO HENRIQUE GOICCALVES MAZZEU}

Doutorando do Departamento de Estatística da Universidad Carlos III de Madrid. Avenida Juan de la Cierva, Getafe, Madri - Espanha - CEP 28903 E-mail: joão.hgm@uol.com.br

\section{NEWTON CARNEIRO AFFONSO DA COSTA JUNIOR}

Doutor pela Escola de Administração de Empresas da Fundação Getulio Vargas (Eaesp-FGV).

Professor do Departamento de Economia e Relações Internacionais da Universidade Federal de Santa Catarina (UFSC). Campus Universitário, Trindade, Florianópolis - SC - Brasil - CEP 88040-900

E-mail:newton@cse.ufsc.br

\section{ANDRÉ ALVES PORTELA SANTOS}

Doutor pelo Departamento de Estatística da Universidad Carlos III de Madrid. Professor do Departamento de Economia e Relações Internacionais da Universidade Federal de Santa Catarina (UFSC).

Campus Universitário, Trindade, Florianópolis - SC - Brasil - CEP 88040-900

E-mail: andre.portela@ufsc.br

Este artigo pode ser copiado, distribuído, exibido, transmitido ou adaptado desde que citados, de forma clara e explícita, o nome da revista, a edição, o ano, e as páginas nas quais o artigo foi publicado originalmente, mas sem sugerir que a RAM endosse a reutilização do artigo. Esse termo de licenciamento deve ser explicitado para os casos de reutilização ou distribuição para terceiros. Não é permitido o uso para fins comerciais. 


\section{RESUMO}

Modelos de precificação de ativos representam uma das áreas mais discutidas e pesquisadas em finanças. São amplamente utilizados de forma teórica e prática na área de investimentos para modelar e prever o risco e o retorno de títulos e de carteiras, bem como em finanças corporativas para estimar o custo de capital e ranquear projetos de investimento. Eles fornecem uma medida útil de risco que ajuda gerentes e investidores a determinar o retorno requerido ao colocarem seu dinheiro em risco. O objetivo deste trabalho é analisar o desempenho do modelo CAPM condicional com aprendizagem proposto por Adrian e Franzoni (2009) quando aplicado às séries de retornos das ações mais líquidas do mercado brasileiro no período de I987 a 2010. Adrian e Franzoni (2009), em seu artigo, complementaram a literatura do CAPM condicional ao modelarem um novo tipo de variação temporal nos betas condicionais. Nesse ambiente, os investidores formam expectativas sobre o nível de longo prazo dos fatores de risco com base nos retornos realizados de variáveis exógenas. Como consequência direta dessa hipótese, os betas condicionais são modelados por meio do filtro de Kalman. Utilizando dados de 25 carteiras classificadas por tamanho e pelo índice valor contábil-valor de mercado, os autores concluíram que o CAPM condicional com aprendizagem é capaz de reduzir substancialmente os erros de apreçamento quando comparado ao CAPM em sua versão original. Dessa forma, contribuímos para a literatura de precificação de ativos, na medida em que avaliamos se esse modelo é capaz de reduzir os erros de apreçamento em relação à versão original do modelo CAPM, quando aplicado a dados de ativos individuais brasileiros. Os resultados deste artigo evidenciam uma redução nos erros de precificação do CAPM condicional com aprendizagem em relação ao CAPM em sua versão original. Dessa forma, tais resultados empíricos sugerem que a aprendizagem sobre os betas deve ser levada em consideração na estimação do CAPM incondicional e condicional. 


\section{PALAVRAS-CHAVE}

CAPM condicional; Filtro de Kalman; Previsão; Coeficiente beta; Erros de apreçamento.

\section{INTRODUÇÃO}

Sharpe (1964), Lintner (1965) e Mossin (1966) introduzem o Capital Asset Pricing Model (CAPM), o qual estabelece um modelo de apreçamento de ativos em um mercado em equilíbrio no qual investidores compartilham as mesmas informações e a mesma função de utilidade. Desde a sua proposição, um grande número de trabalhos tem buscado avaliar seu desempenho em explicar fatos estilizados encontrados nas séries financeiras. Entretanto, estudos empíricos constataram que o CAPM em sua versão original não lograva acomodar, em sua formulação original, uma série de características observadas empiricamente. Fama e French (1992), por exemplo, observaram que o efeito da diferença entre empresas em termos de tamanho e de índice valor contábil-valor de mercado não foi explicado pelo CAPM, confirmando as anomalias denominadas efeito tamanho e efeito valor contábil-valor de mercado.

Desde então, os modelos de apreçamento de ativos têm trazido especificações alternativas no intuito de aprimorar seu desempenho quando aplicados a séries financeiras. Duas vertentes principais surgem a fim de melhorar o desempenho da versão original do CAPM: os modelos multifatores e o CAPM condicional. $\mathrm{O}$ primeiro grupo renuncia à estrutura baseada em um único fator de risco e a estende para um modelo multifator. Nessa linha, Fama e French (I993) sugerem um modelo de três fatores, no qual o prêmio de risco esperado de uma ação deve depender do fator mercado, fator tamanho e fator valor contábil-valor de mercado.

O segundo grupo de pesquisa preserva o modelo de um fator e propõe que o CAPM se sustenta apenas condicionalmente. Em outras palavras, o CAPM deveria utilizar informação condicional como forma de melhorar seu desempenho. Entre as especificações do CAPM condicional, destacam-se as propostas por Jagannathan e Wang (I996) e Lettau e Ludvigson (200I). Em ambos os casos, os autores modelam a evolução da distribuição condicional dos retornos como uma função de variáveis de estado defasadas ${ }^{\mathrm{I}}$. Os autores especificam a

Variáveis de estado, também conhecidas como variáveis condicionantes na literatura do CAPM condicional, são variáveis instrumentais (exógenas) utilizadas para modelar a evolução temporal do beta. Dessa forma, os retornos esperados dos ativos ou carteiras são regredidos em função de um beta condicionado às informações passadas ou correntes. 
covariância entre o retorno de mercado e os retornos das carteiras como funções afins dessas variáveis. Essa especificação é estimada como um modelo multifator, no qual os fatores adicionais são as interações entre o retorno de mercado e as variáveis de estado.

Com base nesses trabalhos, Adrian e Franzoni (2009) complementam a literatura do CAPM condicional ao incorporarem a aprendizagem dos investidores no processo de estimação do beta. Eles assumem que os betas variam ao longo do tempo, seguindo um processo de reversão à média. Além disso, postula-se que os investidores não têm informações sobre o nível de longo prazo do risco sistemático de um dado ativo. Logo, é necessário inferir sobre o nível do beta corrente e de sua média de longo prazo utilizando o histórico dos retornos realizados. Dessa forma, a não observação dos verdadeiros betas levam os investidores a se engajar em um processo de aprendizagem, em que eles atualizam as expectativas sobre os betas à medida que novas informações chegam. Dessa maneira, o risco que determina os retornos esperados é a expectativa do beta que resulta do processo de aprendizagem. Adrian e Franzoni (2009) modelam essa expectativa via filtro de Kalman ${ }^{2}$, no qual o beta é tratado como uma variável latente.

Em linha com esse argumento teórico, Adrian e Franzoni (2009) utilizam o modelo CAPM com aprendizagem para explicar os retornos de 25 carteiras classificadas por tamanho e pelo índice valor contábil-valor de mercado. Os autores mostram que essa versão alternativa do CAPM resulta em uma redução substancial nos erros de apreçamento em comparação ao CAPM incondicional. Além disso, ao introduzirem a aprendizagem no CAPM condicional de Lettau e Ludvigson (200I), os erros são reduzidos significativamente quando comparado ao CAPM incondicional. Adrian e Franzoni (2009) ainda argumentam que o CAPM com aprendizagem é capaz de explicar uma fração do efeito valor. Especificamente, eles mostraram que os betas obtidos pelo filtro de Kalman foram maiores do que as estimativas feitas por meio de mínimos quadrados ordinários (MQO) para as carteiras de valor, o que é crucial para explicar tanto a fração do efeito valor quanto os retornos esperados exigidos pelos investidores, que estão aprendendo sobre o verdadeiro nível do beta.

Além de Adrian e Franzoni (2009), Huang e Hueng (2008) e Trecroci (2009) também levaram em consideração a aprendizagem sobre o beta e sugeri-

${ }^{2}$ O filtro de Kalman consiste em um conjunto de equações matemáticas que fornecem uma solução recursiva ótima para o método de mínimos quadrados. O objetivo dessa solução consiste em calcular um estimador linear, não viesado e ótimo do estado de um sistema em t com base na informação disponível em t-I, e atualizar, com a informação disponível em t, tais estimativas. O filtro trabalha assumindo que o sistema pode ser descrito por meio de um modelo estocástico linear, em que tanto o erro associado ao sistema quanto a informação adicional que se incorpora no modelo têm uma distribuição normal com média zero e variância constante. 
ram um modelo de regressão de parâmetro linear variável no tempo e estimado pelo filtro de Kalman. Huang e Hueng (2009), ao aplicarem os mínimos quadrados adaptativos com fundamentos de Kalman (ALSKF) proposto por McCulloch (2006) para estimar o beta variante no tempo, mostram que o ALSKF melhora a precisão da sua estimativa ao simular o processo de aprendizagem dos investidores com relação ao beta não observável que o modelo OLS não leva em conta. Por fim, Trecroci (2009) mostra que o CAPM com beta variante no tempo apresenta um poder de previsão dos retornos de carteiras sistematicamente superior ao CAPM convencional estimado por OLS.

No Brasil há poucos estudos envolvendo o filtro de Kalman na estimação do CAPM. Silva Filho e Frascaroli (2005), por exemplo, estimaram os betas das ações do setor de telecomunicações brasileiro a partir do modelo de mercado e utilizaram o filtro de Kalman para estimar os efeitos Garch dos resíduos da equação do CAPM e o caminho temporal dos coeficientes do modelo de mercado. Concluíram que o modelo oferece uma boa explicação para o comportamento do setor de telecomunicações no mercado de ações e se mostrou adequado estatisticamente. No entanto, tal estudo não incorpora nenhuma das abordagens de Adrian e Franzoni (2009), Huang e Hueng (2008) ou Trecroci (2009) e não estimam o CAPM condicional.

Portanto, inspirado por Adrian e Franzoni (2009), este artigo aplica o CAPM condicional com aprendizagem no mercado acionário brasileiro com o objetivo de avaliar se esse modelo é capaz de reduzir os erros de apreçamento em comparação ao CAPM incondicional e o condicional sem aprendizagem. O artigo foca a análise do erro de apreçamento de 13 ações com maior índice de liquidez na Bolsa de Valores de São Paulo (Bovespa) entre I987 e 20Io. Os resultados indicam que, quando se considera a aprendizagem sobre o nível do beta, os erros de apreçamento sofrem uma redução quando comparados aos resultados obtidos pelo modelo CAPM condicional e incondicional sem aprendizagem.

Convém ressaltar que testes empíricos da natureza dos pretendidos nesta pesquisa ainda não foram realizados com o modelo CAPM condicional aplicado aos dados do Brasil, o que reflete a importância deste estudo.

Além disso, vale destacar que os resultados deste trabalho não contribuem somente para a literatura do CAPM, mas também podem ser relevantes para empresas, gestores e analistas, na área de investimentos, por meio da análise de risco-retorno e alocação de recursos. Por exemplo, o CAPM é um dos modelos mais utilizados para estimar o custo dos capitais próprios, ou seja, a rentabilidade que os investidores exigem para suportar o risco sistemático de investir na empresa. Dessa forma, uma melhor modelagem do CAPM poderia melhorar a tomada de decições no âmbito financeiro da empresa, contribuindo para sua 
avaliação e para a determinação da estrutura ótima de capitais. Adrian e Franzoni (2004, 2009) argumentam que um modelo econométrico que falha em imitar o processo de aprendizagem dos investidores em relação à evolução temporal do beta pode levar a estimativas incorretas do beta. Por isso, é crucial utilizar instrumentos mais sofisticados de inferência estatística e adotar procedimentos mais rigorosos na especificação do modelo de mercado.

Enfim, espera-se que o trabalho possa avançar na compreensão do CAPM, o que reflete na busca de uma melhor medida de risco sistemático e de uma relação mais próxima entre mercado acionário e comportamento dos agentes.

O artigo está organizado da seguinte forma: a Seção 2 introduz o CAPM condicional com aprendizagem de Adrian e Franzoni (2009), a Seção 3 analisa os resultados da estimação dos parâmetros do modelo via filtro de Kalman, a Seção 4 apresenta a metodologia do cálculo dos erros de apreçamento e compara o desempenho do CAPM condicional com aprendizagem com outras especificações do CAPM, e, finalmente, a Seção 5 apresenta as considerações finais.

\section{CAPM CONDICIONAL COM APRENDIZAGEM}

O CAPM condicional com aprendizagem de Adrian e Franzoni (2009) é uma variação do CAPM condicional baseada na premissa de que os investidores devem formar expectativas sobre o nível de risco do ativo ao longo do tempo. Para tanto, os autores adicionam à especificação do CAPM condicional uma dinâmica para o beta do ativo. Com a hipótese de não arbitragem e parâmetros desconhecidos do processo dos retornos, Adrian e Franzoni (2009) propõem uma especificação na qual o CAPM condicional é assegurado. Formalmente, o modelo é escrito como:

$$
E_{t}\left[R_{t+1}^{i}\right]=\beta_{t+\mathrm{I} \mid t}^{i s} E_{t}\left[R_{t+\mathrm{I}}^{M}\right]
$$

em que $R_{t+\mathrm{I}}^{i}$ é o excesso de retorno do ativo $i$, e $\beta_{t+\mathrm{I} \mid t}^{i s}=E_{t}\left[\beta_{t+\mathrm{I}}^{i}\right]=\operatorname{cov}_{t}\left(R_{t+\mathrm{I}}^{i}, R_{t+\mathrm{I}}^{M}\right) /$ $\operatorname{var}_{t}\left(R_{t+1}^{M}\right)$ é, ao mesmo tempo, a expectativa no tempo $t$ do fator de risco que se aplica aos retornos em $t+$ I e o beta condicional. Os autores assumem que $\beta_{t+\mathrm{I}}^{i}$ 148 segue um processo autorregressivo condicionado a um vetor de variáveis exógenas e estacionárias $\left(\gamma_{t}\right)$ :

$$
\beta_{t+1}^{i}=\left(\mathrm{I}-F^{i}\right) B^{i}+F^{i} \beta_{t}^{i}+\varnothing^{i^{\prime}} \gamma_{t}+u_{t+1}^{i}
$$


em que Ø é um vetor de parâmetros associados às variáveis exógenas. Assumindo que a média das variáveis condicionantes $\gamma_{t}$ é zero ao longo do tempo, podemos interpretar $B^{i}$ como a média de longo prazo do fator de risco $\beta_{t+1}^{i}$. Dessa forma, ao descartarmos as variáveis exógenas $\gamma_{\mathrm{t}}$, o modelo resultante é o CAPM com fator de aprendizagem dado pela evolução temporal do parâmetro $\beta_{t+1}^{i}$. Assumimos que sua média de longo prazo não seja observável, de modo que os investidores tenham que formar expectativas sobre o nível corrente de risco do ativo $i$, $\beta_{t+1}^{i}$, e sobre o nível de risco de longo prazo, $B^{i}$. É importante notar que, apesar de $B^{i}$ e $F^{i}$ não terem o tempo em subscrito, apenas o parâmetro autorregressivo $F^{i}$ é invariante no tempo; o beta de longo prazo $B^{i}$, por sua vez, é o objeto de aprendizagem, e, por isso, sua estimativa é atualizada constantemente à medida que novas informações chegam. Finalmente, $u_{t+1}^{i}$ é um choque puramente idiossincrático. Logo, as inovações dos fatores de risco são não correlacionadas com o prêmio de risco.

Seguindo a expressão para os retornos em excesso proposta por Adrian e Franzoni (2009), temos:

$$
R_{t+1}^{i}=\beta_{t+1}^{i} R_{t+1}^{M}+\eta_{t+1}
$$

em que $\eta_{t+\mathrm{I}}=\left(\beta_{t+\mathrm{I} \mid t}^{i s}-\beta_{t+\mathrm{I}}^{i}\right) E_{t}\left[R_{t+\mathrm{I}}^{M}\right]+\varepsilon_{t+\mathrm{I}}^{i}$, sendo $\eta_{t+\mathrm{I}}$ ortogonal a $R_{t+\mathrm{I}}^{M}$ e $\beta_{t+\mathrm{I} \mid t}^{i s}=$ $E_{t}\left[\beta_{t+1}^{i}\right]$. Esse resultado é uma condição necessária para a aplicação do filtro de Kalman na estimativa de $\beta_{t+1}^{i}$ na Equação (3). Além disso, assume-se que os choques $u_{t+1}^{i}$ e $\eta_{t+1}^{i}$ são condicionalmente normais. Portanto, a expectativa condicional de $\beta_{t+\mathrm{I}}^{i}$ se desenvolve de acordo com o filtro de Kalman:

$$
\beta_{t+\mathrm{I} \mid t}^{i s}=\left(\mathrm{I}-F^{i}\right) B_{t-\mathrm{I}}^{i s}+F^{i} \beta_{t \mid t-\mathrm{I}}^{i s}+\varnothing^{i^{i}} Y_{t}+k_{t}^{i}\left(R_{t}^{i}-E_{t-\mathrm{I}}\left[R_{t}^{i}\right]\right)
$$

em que $B_{t-\mathrm{I}}^{i s}=E_{t-\mathrm{I}}\left[B^{i}\right]$ e $\beta_{t \mid t-\mathrm{I}}^{i s}=E_{t-\mathrm{I}}\left[B_{t}^{i}\right] . k_{t}^{i}$ é mais conhecido como "ganho" de Kalman e pode ser interpretado como um coeficiente de regressão variante no tempo. A regra ótima é utilizar a parte não esperada da realização do retorno corrente para atualizar a estimativa do próximo período do fator de risco $\beta_{t}^{i}$. Nesse sentido, a Equação (4) mostra que a previsão de um período à frente do fator de risco é uma combinação do comportamento de longo prazo de $\beta_{t}^{i}$, capturado pela

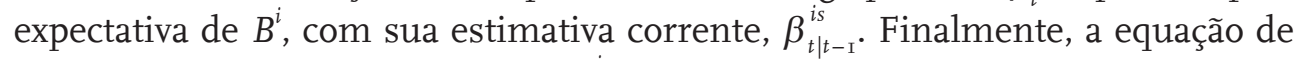
atualização para as expectativas de $B^{i}$ é

$$
B_{t}^{i s}=B_{t-1}^{i s}+K_{t}^{i}\left(R_{t}^{i}-E_{t-1}\left[R_{t}^{i}\right]\right)
$$


A Equação (5) indica que, embora a média de longo prazo do beta seja constante, suas expectativas variam ao longo do tempo. Além disso, a equação implica que $B_{t}^{i s}$ segue um processo puramente aleatório, sendo, então, passível de ser estimado recursivamente. Essa característica introduz um componente móvel lento nos fatores de risco e nos prêmios de risco exigidos. Em suma, o CAPM condicional com aprendizagem é representado pelo seguinte modelo estado-espaço ${ }^{3}$ :

$$
\begin{gathered}
R_{t+\mathrm{I}}^{i}=\beta_{t+\mathrm{I}}^{i} R_{t+\mathrm{I}}^{M}+\eta_{t+\mathrm{I}}^{i} \\
\beta_{t+\mathrm{I}}^{i}=\left(\mathrm{I}-F^{i}\right) B^{i}+F^{i} \beta_{t}^{i}+\varnothing^{i^{\prime}} Y_{t}+u_{t+\mathrm{I}}^{i} \\
B^{i} \text { : não observável. }
\end{gathered}
$$

Assume-se que os termos de erro, $\eta_{t+1}^{i}$ e $u_{t+1}^{i}$, seguem uma distribuição normal e que esses erros não são correlacionados entre si e entre diferentes períodos de tempo. O parâmetro autorregressivo $F^{i}$, as variâncias dos termos de erro $\left(\sigma_{\eta}^{i}\right)^{2} \mathrm{e}$ $\left(\sigma_{u}^{i}\right)^{2}$ e os parâmetros das variáveis condicionantes $\varnothing^{i}$ são estimados por máxima verossimilhança. Utilizamos como condição inicial o valor de I para os valores iniciais de $\beta^{i}$ e $B^{i}$ e o procedimento de inicialização difusa como condições iniciais para as variâncias de $\beta^{i}$ e $B^{i}$. A derivação do filtro de Kalman pode ser encontrada em detalhes em Adrian e Franzoni (2009). A técnica de estimação por máxima verossimilhança adotada está detalhada em Koopman e Durbin (200I).

No entanto, para entender de forma mais clara a contribuição desse modelo em relação aos conhecidos modelos condicionais do CAPM, como os de Jagan-

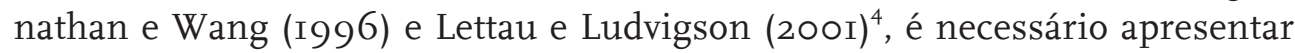
suas estruturas básicas. Tambosi Filho e Garcia (2007), ao fazerem um estudo sobre os testes do CAPM condicional, apresentaram de forma geral as principais equações desse modelo.

$$
E\left[R_{i t} \mid I_{t-\mathrm{I}}\right]=\gamma_{o t-\mathrm{I}}+\gamma_{\mathrm{It}-\mathrm{I}} \beta_{i t-\mathrm{I}}
$$

em que $I_{t-1}$ é o nível de informação dos investidores no final do período t-I, $\gamma_{o t-1}$ é o retorno esperado condicional sobre o portfólio com beta igual a zero, $\gamma_{\mathrm{It-I}}$ é o

A derivação do filtro de Kalman associado ao modelo CAPM com aprendizagem está detalhada no Apêndice B deste artigo.

4 Adrian e Franzoni (2009) mostram que essa abordagem é equivalente a uma regressão temporal de um modelo multifator, no qual os fatores adicionais são as interações entre o retorno de mercado e as variáveis de condicionantes. Esse modelo é apresentado na Equação io. 
prêmio de risco de mercado condicional, e $\beta_{i t-\mathrm{I}}$ é beta condicional do ativo i no período t-I definido como:

$$
\beta_{i t-\mathrm{I}}=\operatorname{Cov}\left(R_{i t}, R_{m t} \mid I_{t-\mathrm{I}}\right) / \operatorname{Var}\left(R_{m t} \mid I_{t-\mathrm{I}}\right)
$$

As equações 9 e Io são utilizadas por Jagannathan e Wang (I996) para explicar a variação cross-sectional do retorno esperado condicional em diferentes ativos. O modelo condicional é uma forma conveniente para incorporar variâncias e covariâncias que se modificam ao longo do tempo, que, por sua vez, são condicionadas às variáveis de informação (condicionantes) defasadas $I_{t-1}$. O trabalho de Lettau e Ludvigson (200I) se destaca por incluir a variável razão entre consumo agregado e riqueza no conjunto de informação $I_{t-1}$, mais conhecida como CAY (consumption-to-wealth ratio). Segundo os autores, essa variável captura as inovações da cointegração entre o consumo, o mercado de ações e a renda do trabalho. Lettau e Ludvigson (200I) mostram que a CAY prevê o prêmio de risco e que, quando usada como variável condicionante, melhora o desempenho de apreçamento do CAPM condicional em relação ao CAPM incondicional.

Portanto, fica possível entender a contribuição do modelo de Adrian e Franzoni (2009) ao CAPM condicional, pois, ao estimarem o beta como uma função de variáveis exógenas via filtro de Kalman, eles inovam a especificação do CAPM condicional ao adicionarem uma dinâmica para o beta do ativo, o qual é estimado e atualizado recursivamente, refletindo a premissa de que os investidores devem formar expectativas sobre o nível de risco do ativo à medida que novas informações são disponíveis.

\section{DADOS UTILIZADOS E RESULTADOS DA ESTIMAÇÃO}

O presente trabalho tem como objetivo comparar os erros de apreçamento entre o CAPM condicional com aprendizagem e o CAPM incondicional e condicional estimados via MQO. Para alcançar esse objetivo, foram escolhidas 30 ações mais líquidas do mercado brasileiro durante o intervalo de I987 a 20 Io. Foi utilizado o índice de liquidez médio anual de cada ação, coletado em 3 I de dezembro de cada ano para a seleção das ações. Em seguida, calculou-se a média da série do índice de liquidez anual de cada ação de I987 a 20I0, e as ações foram classificadas em ordem decrescente em relação à média de seu respectivo índice de liquidez. Foi obtido, dessa forma, um ranking das ações mais líquidas. Dentre as 30 ações com o maior índice de liquidez, foram excluídas aquelas que não 
apresentaram cotações de julho de I987 a julho de 20I0, restando, portanto, I3 ações para o presente estudo: PETR4, VALE5, $\mathrm{BBDC}_{4}, \mathrm{CMIG}_{4}, \mathrm{ITUB}_{4}, \mathrm{TLPP}_{3}$, $\mathrm{PETR}_{3}, \mathrm{AMBV}_{4}, \mathrm{ITSA}_{4}, \mathrm{BRKM}_{5}, \mathrm{VALE}_{3}, \mathrm{FIBR}_{3}, \mathrm{BBAS}_{3}^{5}$.

Seguindo os trabalhos de Campbell e Vuolteenaho (2004) e Adrian e Franzoni (2009), foram selecionadas as seguintes variáveis condicionantes: o excesso de retorno do mercado (PRM), o spread de juros (SPREAD) e o spread de valor (value spread) (HML). O excesso de retorno do mercado foi construído pela diferença entre o retorno do Ibovespa e o retorno da taxa Selic, utilizada como proxy do ativo livre de risco. O spread de juros foi construído pela diferença entre a taxa Selic e a taxa CDI. Finalmente, o spread de valor foi construído com base na diferença entre o retorno de uma carteira formada por ações de valor e o retorno de uma carteira formada por ações de crescimento ${ }^{6}$. Esta variável é comumente conhecida na literatura financeira pelo fator de risco HML (high value minus low value), introduzido por Fama e French (I993) . Todas as variáveis utilizadas foram coletadas na frequência semanal entre io de julho de I987 e 2 de julho de 20 Io, totalizando I.200 observações de cada variável. Os dados provêm da base de dados Economática e foram deflacionados pelo índice de preços IGP-DI. A Tabela I reporta estatísticas descritivas de todas as variáveis utilizadas neste artigo.

\section{TABELA I}

ESTATISTICA DESCRITIVA DAS VARIÁVEIS UTILIZADAS NO ARTIGO*

\begin{tabular}{l|c|c|c|c|c|c|c|c|c|c}
\hline \multicolumn{10}{|c}{ PAINEL A: AÇÕES } \\
\hline ATIVOS & MÉDIA & MEDIANA & MÁXIMO & MÍNIMO & $\begin{array}{c}\text { DESVIO- } \\
\text {-PADRÃO }\end{array}$ & ASSIMETRIA & CURTOSE & JARQUE-BERA & PROB. & $\begin{array}{c}\text { NÚMERO DE } \\
\text { OBSERVAÇÕES }\end{array}$ \\
\hline PETR4 & 0,10 & 0,36 & 50,35 & $-103,32$ & 9,07 & $-1,51$ & 22,18 & 18845,720 & 0,000 & 1200 \\
\hline VALE5 & 0,18 & 0,30 & 44,68 & $-83,18$ & 8,05 & $-0,93$ & 15,47 & 7946,751 & 0,000 & 1200 \\
\hline BBDC4 & 0,17 & $-0,19$ & 42,62 & $-57,64$ & 7,85 & $-0,12$ & 7,38 & 961,282 & 0,000 & 1200 \\
\hline CMIG4 & 0,07 & $-0,22$ & 96,33 & $-75,95$ & 10,39 & 0,68 & 14,96 & 7250,366 & 0,000 & 1200 \\
\hline
\end{tabular}

(continua)

5 Pode-se argumentar que a escolha das ações por meio do índice de liquidez pode induzir ao problema do viés de sobrevivência. Entretanto, a análise do erro de apreçamento continua válida para quaisquer tipos de ativos, tanto aqueles selecionados com ou sem viés de sobrevivência.

6 Para maiores detalhes sobre a construção das duas carteiras, ver o Apêndice A do artigo.

7 Também testamos outra variável condicionante na equação do beta condicional, a taxa de câmbio Ptax real/dólar. No entanto, essa variável piorou o desempenho tanto do CAPM condicional com aprendizagem quanto do CAPM condicional (estimado por MQO), e, por isso, não reportamos seus resultados. 


\section{TABELA I (CONCLUSÃO)}

\section{ESTATISTICA DESCRITIVA DAS VARIÁVEIS}

UTILIZADAS NO ARTIGO*

\begin{tabular}{|c|c|c|c|c|c|c|c|c|c|c|}
\hline \multicolumn{11}{|c|}{ PAINEL A:AÇÕES } \\
\hline ATIVOS & MÉDIA & MEDIANA & MÁXIMO & MÍNIMO & $\begin{array}{l}\text { DESVIO- } \\
\text {-PADRÃO }\end{array}$ & ASSIMETRIA & CURTOSE & JARQUE-BERA & PROB. & $\begin{array}{l}\text { NÚMERO DE } \\
\text { OBSERVAÇÕES }\end{array}$ \\
\hline ITUB4 & 0,24 & 0,02 & 39,79 & $-107,14$ & 8,33 & $-1,54$ & 27,13 & 29594,720 & 0,000 & 1200 \\
\hline TLPP3 & 0,17 & $-0,27$ & 63,68 & $-51,98$ & 9,57 & 0,65 & 8,63 & 1661,870 & 0,000 & 1196 \\
\hline PETR3 & 0,15 & $-0,02$ & 41,66 & $-47,23$ & 8,81 & 0,17 & 6,90 & 758,773 & 0,000 & 1188 \\
\hline AMBV4 & 0,18 & 0,28 & 63,13 & $-69,03$ & 7,56 & $-0,06$ & 14,76 & 6913,574 & 0,000 & 1200 \\
\hline ITSA4 & 0,20 & $-0,02$ & 38,77 & $-91,34$ & 8,15 & $-0,87$ & 18,04 & 11465,800 & 0,000 & 1200 \\
\hline BRKM5 & $-0,11$ & $-0,33$ & 42,23 & $-77,79$ & 8,63 & $-0,26$ & 9,91 & 2399,150 & 0,000 & 1200 \\
\hline VALE3 & 0,30 & $-0,05$ & 49,54 & $-52,54$ & 8,35 & 0,14 & 7,92 & 1202,556 & 0,000 & 1188 \\
\hline FIBR3 & $-0,11$ & $-0,42$ & 58,02 & $-62,77$ & 8,65 & 0,22 & 9,07 & 1848,752 & 0,000 & 1200 \\
\hline BBAS3 & $-0,04$ & $-0,33$ & 61,38 & $-43,07$ & 8,75 & 0,45 & 7,05 & 859,440 & 0,000 & 1198 \\
\hline \multicolumn{11}{|c|}{ PAINEL B: VARIÁVEIS EXÓGENAS } \\
\hline PRM & 0,02 & 0,39 & 34,54 & $-71,14$ & 6,94 & $-1,21$ & 15,10 & 7611,663 & 0,000 & 1200 \\
\hline HML & 0,25 & 0,17 & 21,61 & $-39,59$ & 4,57 & $-0,35$ & 9,38 & 2059,692 & 0,000 & 1200 \\
\hline SPREAD & $-0,03$ & 0,00 & 1,95 & $-2,90$ & 0,22 & $-3,86$ & 61,44 & 173729,4 & 0,000 & 1200 \\
\hline
\end{tabular}

* O painel A apresenta as estatísticas das I3 ações estudadas, o número de observações varia de I.200 (para a maioria das ações) a I.I88 (ação PETR3). O painel B apresenta as estatísticas das variáveis exógenas utilizadas. Os dados são semanais compreendendo o período: 10/07/1987 02/07/2010.

PRM: excesso de retorno do mercado, construído pela diferença entre o retorno do Ibovespa e o retorno da taxa Selic. HML: spread de diferença entre o retorno de uma carteira formada por ações de valor e o retorno de uma carteira formada por ações de crescimento. SPREAD: spread de juros, construído pela diferença entre a taxa Selic e a taxa CDI.

Fonte: Elaborada pelos autores.

O modelo de espaço de estados dados pelas equações (6) a (8) foi estimado por máxima verossimilhança utilizando todos os dados disponíveis na amostra para cada uma das ações. Os parâmetros estimados e seus respectivos p-valores estão reportados na Tabela 2. Duas especificações alternativas do modelo CAPM 
com aprendizagem foram estimadas. Na primeira especificação (Painel A), nenhuma variável condicionante foi incluída. A segunda especificação (Painel B) utiliza as variáveis condicionantes de Campbell e Vuolteenaho (2004): o retorno do mercado defasado, o spread de valor e o spread de juros, denotados por $M K T$, HML e TERM, respectivamente.

\section{TABELA 2}

ESTIMATIVAS POR MÁXIMA VEROSSIMILHANÇA DOS PARÂMETROS DO MODELO CAPM COM APRENDIZAGEM (EQUAÇÕES 6 A 8)*

\begin{tabular}{|c|c|c|c|c|c|c|c|c|c|c|c|c|c|}
\hline & PETR4 & VALE5 & BBDC4 & CMIG4 & ITUB4 & TLPP3 & PETR3 & AMBV4 & ITSA4 & BRKM5 & VALE3 & FIBR3 & BBAS3 \\
\hline \multicolumn{14}{|c|}{ PAINEL A: CAPM INCONDICIONAL COM APRENDIZAGEM } \\
\hline$F$ & $\begin{array}{c}0,0953 \\
{[0,3894]}\end{array}$ & $\begin{array}{c}0,9748 \\
{[0,0000]}\end{array}$ & $\begin{array}{c}-0,0680 \\
{[0,4220]}\end{array}$ & $\begin{array}{c}0,3983 \\
{[0,0000]}\end{array}$ & $\begin{array}{c}0,0066 \\
{[0,9232]}\end{array}$ & $\begin{array}{c}0,1676 \\
{[0,0194]}\end{array}$ & $\begin{array}{c}0,1273 \\
{[0,0546]}\end{array}$ & $\begin{array}{c}-0,0138 \\
{[0,8090]}\end{array}$ & $\begin{array}{c}0,1378 \\
{[0,0592]}\end{array}$ & $\begin{array}{c}-0,0450 \\
{[0,5175]}\end{array}$ & $\begin{array}{c}0,0691 \\
{[0,5056]}\end{array}$ & $\begin{array}{c}0,0912 \\
{[0,2111]}\end{array}$ & $\begin{array}{l}-0,0286 \\
{[0,6300]}\end{array}$ \\
\hline$\sigma_{n}^{2}$ & $\begin{array}{l}17.3883 \\
{[0,0000]}\end{array}$ & $\begin{array}{l}24.1721 \\
{[0,0000]}\end{array}$ & $\begin{array}{l}20.9681 \\
{[0,0000]}\end{array}$ & $\begin{array}{l}33.5790 \\
{[0,0000]}\end{array}$ & $\begin{array}{l}21.2191 \\
{[0,0000]}\end{array}$ & $\begin{array}{l}36.3575 \\
{[0,0000]}\end{array}$ & $\begin{array}{l}34.5152 \\
{[0,0000]}\end{array}$ & $\begin{array}{l}20.2894 \\
{[0,0000]}\end{array}$ & $\begin{array}{l}19.8241 \\
{[0,0000]}\end{array}$ & $\begin{array}{l}35.2688 \\
{[0,0000]}\end{array}$ & $\begin{array}{l}38.5671 \\
{[0,0000]}\end{array}$ & $\begin{array}{l}32.2785 \\
{[0,0000]}\end{array}$ & $\begin{array}{l}35.7089 \\
{[0,0000]}\end{array}$ \\
\hline$\sigma_{u}^{2}$ & $\begin{array}{c}0.1613 \\
{[0,0000]}\end{array}$ & $\begin{array}{c}0.0063 \\
{[0,0000]}\end{array}$ & $\begin{array}{c}0.2875 \\
{[0,0000]}\end{array}$ & $\begin{array}{c}0.4028 \\
{[0,0000]}\end{array}$ & $\begin{array}{c}0.4883 \\
{[0,0000]}\end{array}$ & $\begin{array}{c}0.4596 \\
{[0,0000]}\end{array}$ & $\begin{array}{c}0.3621 \\
{[0,0000]}\end{array}$ & $\left.\begin{array}{c}0.2998 \\
{[0,0000]}\end{array}\right]$ & $\left.\mid \begin{array}{c}0.3471 \\
{[0,0000]}\end{array}\right]$ & $\begin{array}{c}0.1477 \\
{[0,0000]}\end{array}$ & $\begin{array}{c}0.2771 \\
{[0,0000]}\end{array}$ & $\begin{array}{c}0.4101 \\
{[0,0000]}\end{array}$ & $\begin{array}{c}0.3369 \\
{[0,0000]}\end{array}$ \\
\hline \multicolumn{14}{|c|}{ PAINEL B: CAPM CONDICIONAL COM APRENDIZAGEM. VARIÁVEIS CONDICIONANTES: MKT, HML E TERM } \\
\hline$F$ & $\begin{array}{c}0,1358 \\
{[0,2099]}\end{array}$ & $\begin{array}{c}0,9739 \\
{[0,0000]}\end{array}$ & $\begin{array}{c}-0,0536 \\
{[0,5379]}\end{array}$ & $\begin{array}{c}0,4206 \\
{[0,0000]}\end{array}$ & $\begin{array}{c}-0,0269 \\
{[0,7079]}\end{array}$ & $\begin{array}{c}0,1675 \\
{[0,0308]}\end{array}$ & $\begin{array}{c}0,1436 \\
{[0,0409]}\end{array}$ & $\begin{array}{c}-0,0546 \\
{[0,5375]}\end{array}$ & $\begin{array}{c}0,1385 \\
{[0,0681]}\end{array}$ & $\begin{array}{c}-0,1628 \\
{[0,2364]}\end{array}$ & $\begin{array}{c}0,1764 \\
{[0,0969]}\end{array}$ & $\begin{array}{c}0,2187 \\
{[0,0285]}\end{array}$ & $\begin{array}{l}-0,0507 \\
{[0,5602]}\end{array}$ \\
\hline$\Phi^{M K T}$ & $\begin{array}{c}-0,0053 \\
{[0,1088]}\end{array}$ & $\begin{array}{c}0,0025 \\
{[0,0210]}\end{array}$ & $\begin{array}{c}-0,0011 \\
{[0,7694]}\end{array}$ & \begin{tabular}{|c|}
$-0,0099$ \\
{$[0,0254]$}
\end{tabular} & $\begin{array}{c}0,0037 \\
{[0,4354]}\end{array}$ & $\begin{array}{c}0,0037 \\
{[0,3047]}\end{array}$ & $\begin{array}{c}-0,0002 \\
{[0,9734]}\end{array}$ & $\begin{array}{c}0,0002 \\
{[0,9473]}\end{array}$ & $\mid \begin{array}{c}0,0021 \\
{[0,5868]}\end{array}$ & $\begin{array}{c}0,0033 \\
{[0,4831]}\end{array}$ & $\begin{array}{c}0,0026 \\
{[0,5801]}\end{array}$ & $\begin{array}{c}0,0016 \\
{[0,7686]}\end{array}$ & $\begin{array}{l}-0,0021 \\
{[0,6676]}\end{array}$ \\
\hline$\Phi^{H M L}$ & $\begin{array}{c}-0,0031 \\
{[0,4274]}\end{array}$ & $\begin{array}{c}-0,0005 \\
{[0,7509]}\end{array}$ & $\begin{array}{c}0,0077 \\
{[0,1480]}\end{array}$ & $\begin{array}{c}0,0041 \\
{[0,4790]}\end{array}$ & $\begin{array}{c}0,0120 \\
{[0,0609]}\end{array}$ & $\begin{array}{c}0,0116 \\
{[0,1079]}\end{array}$ & $\begin{array}{c}-0,0147 \\
{[0,0675]}\end{array}$ & $\begin{array}{c}-0,0085 \\
{[0,1277]}\end{array}$ & $\begin{array}{c}0,0031 \\
{[0,5851]}\end{array}$ & $\begin{array}{c}0,0004 \\
{[0,9532]}\end{array}$ & $\begin{array}{c}-0,0208 \\
{[0,0059]}\end{array}$ & $\begin{array}{c}0,0025 \\
{[0,7370]}\end{array}$ & $\begin{array}{c}0,0058 \\
{[0,4181]}\end{array}$ \\
\hline$\Phi^{\text {TERM }}$ & $\begin{array}{c}0,0455 \\
{[0,5116]}\end{array}$ & $\begin{array}{c}-0,0244 \\
{[0,3628]}\end{array}$ & $\mid \begin{array}{c}0,1369 \\
{[0,1334]}\end{array}$ & \begin{tabular}{|c|}
$-0,1479$ \\
{$[0,2349]$}
\end{tabular} & $\begin{array}{c}-0,0762 \\
{[0,4925]}\end{array}$ & $\begin{array}{c}-0,0655 \\
{[0,5873]}\end{array}$ & $\begin{array}{c}0,0520 \\
{[0,6720]}\end{array}$ & $\begin{array}{c}-0,1967 \\
{[0,1273]}\end{array}$ & $\mid \begin{array}{c}0,0071 \\
{[0,9607]}\end{array}$ & $\begin{array}{c}-0,0511 \\
{[0,5560]}\end{array}$ & $\begin{array}{c}-0,1218 \\
{[0,5226]}\end{array}$ & $\begin{array}{c}-0,3006 \\
{[0,0555]}\end{array}$ & $\begin{array}{c}0,0448 \\
{[0,8030]}\end{array}$ \\
\hline$\sigma_{n}^{2}$ & $\begin{array}{l}17.3970 \\
{[0,0000]}\end{array}$ & $\begin{array}{l}24.1939 \\
{[0,0000]}\end{array}$ & $\begin{array}{l}20.8364 \\
{[0,0000]}\end{array}$ & $\begin{array}{l}33.6731 \\
{[0,0000]}\end{array}$ & $\begin{array}{l}21.2212 \\
{[0,0000]}\end{array}$ & $\begin{array}{l}36.3248 \\
{[0,0000]}\end{array}$ & $\begin{array}{l}34.3259 \\
{[0,0000]}\end{array}$ & $\begin{array}{l}20.2529 \\
{[0,0000]}\end{array}$ & $\begin{array}{c}19.8122 \\
{[0,0000]}\end{array}$ & $\begin{array}{l}35.2230 \\
{[0,0000]}\end{array}$ & $\begin{array}{l}38.7449 \\
{[0,0000]}\end{array}$ & $\begin{array}{l}32.3981 \\
{[0,0000]}\end{array}$ & $\begin{array}{l}35.6340 \\
{[0,0000]}\end{array}$ \\
\hline$\sigma_{u}^{2}$ & $\begin{array}{c}0.1569 \\
{[0,0000]}\end{array}$ & $\begin{array}{c}0.0056 \\
{[0,0000]}\end{array}$ & $\begin{array}{c}0.2904 \\
{[0,0000]}\end{array}$ & $\begin{array}{c}0.3837 \\
{[0,0000]}\end{array}$ & $\begin{array}{c}0.4816 \\
{[0,0000]}\end{array}$ & $\begin{array}{c}0.4574 \\
{[0,0000]}\end{array}$ & $\begin{array}{c}0.3643 \\
{[0,0000]}\end{array}$ & $\begin{array}{c}0.2971 \\
{[0,0000]}\end{array}$ & $\begin{array}{c}0.3479 \\
{[0,0000]}\end{array}$ & $\begin{array}{c}0.1451 \\
{[0,0000]}\end{array}$ & $\begin{array}{c}0.2530 \\
{[0,0000]}\end{array}$ & $\begin{array}{c}0.3842 \\
{[0,0000]}\end{array}$ & $\begin{array}{c}0.3399 \\
{[0,0000]}\end{array}$ \\
\hline
\end{tabular}

* O modelo é estimado utilizando toda a amostra (I0.7.I987-2.7.20I0). No painel A, os parâmetros estimados são o coeficiente autorregressivo $F$, a variância do erro da equação de observação $\sigma_{n}^{2}$ e a variância do erro da equação de estado $\sigma_{u}^{2}$. No painel $\mathrm{B}$, os parâmetros adicionais estimados são o coeficiente do retorno de mercado defasado $\Phi^{M K T}$, o coeficiente do spread de valor $\Phi^{H M L}$ e o coeficiente do spread de juros $\Phi^{T E R M}$.

p-valores estão em colchetes.

Fonte: Elaborada pelos autores. 
Vale observar na Tabela 2 que o coeficiente autorregressivo $F^{i}$ varia razoavelmente entre as diferentes especificações do modelo com aprendizagem, sugerindo que variáveis condicionantes capturam parte da persistência dos betas. Em geral, encontramos que a persistência nos betas é maior na versão condicional que na versão incondicional. Por exemplo, no caso da ação PETR4, o coeficiente autorregressivo $F^{i}$ na versão incondicional é igual a o,0953, enquanto, na versão condicional, é igual a 0,1358. A estimativa da variância idiossincrática do retorno $\left(\sigma_{n}^{i}\right)^{2}$ varia muito pouco entre as diferentes especificações e entre as ações, enquanto a estimativa da variância idiossincrática do beta $\left(\sigma_{u}^{i}\right)^{2}$ varia pouco entre as diferentes especificações, mas varia substancialmente entre as diferentes ações. Note que $\left(\sigma_{n}^{i}\right)^{2}$ e $\left(\sigma_{u}^{i}\right)^{2}$ são significativas a I\% de significância para todas as ações em cada modelo. Com relação às variáveis condicionantes, observa-se que cada uma delas é significativa em pelo menos uma das i3 ações estudadas em cada especificação.

Por fim, a Tabela 3 apresenta um resumo dos betas estimados por MQO (versão original do CAPM) e pelo modelo CAPM com aprendizagem. No caso do CAPM estimado por MQO, o beta é dado pelo coeficiente do retorno do mercado, estimado sobre a amostra inteira. Para o CAPM com aprendizagem, o beta é o obtido pela média da série das previsões do beta de um período à frente construídas a partir do filtro de Kalman. Observa-se que as quatro especificações do CAPM apresentaram valores distintos para os betas de cada um dos ativos.

\section{TABELA 3}

BETAS ESTIMADOS*

\begin{tabular}{lcccc}
\hline & PAINELA & PAINEL B & PAINEL C & PAINEL D \\
\hline AÇÕES & $\begin{array}{c}\text { CAPM } \\
\text { INCONDICIONAL }\end{array}$ & $\begin{array}{c}\text { CAPM } \\
\text { INCONDICIONAL }\end{array}$ & $\begin{array}{c}\text { CAPM CONDICIONAL: } \\
\text { MKT, HML E TERM } \\
\text { DEFASADAS }\end{array}$ & $\begin{array}{c}\text { CAPM CONDICIONAL } \\
\text { COM APRENDIZAGEM: } \\
\text { MKT DEFASADA, HML E } \\
\text { TERM }\end{array}$ \\
\hline PETR4 & $1,0906(0,0209)$ & $1,0453(0,0014)$ & $1,0948(0,0218)$ & $1,0443(0,0023)$ \\
\hline VALE5 & $0,8618(0,0225)$ & $0,8318(0,0093)$ & $0,8620(0,0234)$ & $0,8253(0,0088)$ \\
\hline BBDC4 & $0,7798(0,0237)$ & $0,7511(0,0022)$ & $0,7657(0,0248)$ & $0,7517(0,0024)$ \\
\hline CMIG4 & $1,0311(0,0314)$ & $1,0291(0,0056)$ & $1,0400(0,0328)$ & $1,0124(0,0069)$ \\
\hline ITUB4 & $0,7536(0,0270)$ & $0,6514(0,0025)$ & $0,7811(0,0279)$ & $0,6510(0,0033)$ \\
\hline
\end{tabular}


BETAS ESTIMADOS*

\begin{tabular}{lcccc}
\hline & PAINELA & PAINEL B & PAINEL C & PAINEL D \\
\hline AÇÕES & $\begin{array}{c}\text { CAPM } \\
\text { INCONDICIONAL }\end{array}$ & $\begin{array}{c}\text { CAPM } \\
\text { INCONDICIONAL }\end{array}$ & $\begin{array}{c}\text { CAPM CONDICIONAL: } \\
\text { MKT, HML E TERM } \\
\text { DEFASADAS }\end{array}$ & $\begin{array}{c}\text { CAPM CONDICIONAL } \\
\text { COMPRENDIZAGEM: } \\
\text { MKT DEFASADA, HML E } \\
\text { TERM }\end{array}$ \\
\hline TLPP3 & $0,8893(0,0334)$ & $0,8976(0,0043)$ & $0,9359(0,0344)$ & $0,9020(0,0048)$ \\
\hline PETR3 & $0,8334(0,0310)$ & $0,7962(0,0042)$ & $0,8418(0,0320)$ & $0,7966(0,0048)$ \\
\hline AMBV4 & $0,7151(0,0238)$ & $0,6953(0,0014)$ & $0,6833(0,0246)$ & $0,6858(0,0024)$ \\
\hline ITSA4 & $0,8303(0,0241)$ & $0,7737(0,0033)$ & $0,8383(0,0251)$ & $0,7796(0,0053)$ \\
\hline BRKM5 & $0,8287(0,0268)$ & $0,8492(0,0016)$ & $0,8342(0,0280)$ & $0,8525(0,0034)$ \\
\hline VALE3 & $0,7065(0,0315)$ & $0,7383(0,0036)$ & $0,7071(0,0323)$ & $0,7194(0,0054)$ \\
\hline FIBR3 & $0,7554(0,0287)$ & $0,7421(0,0042)$ & $0,7381(0,0300)$ & $0,7298(0,0061)$ \\
\hline BBAS3 & $0,8012(0,0307)$ & $0,7485(0,0021)$ & $0,8009(0,0320)$ & $0,7580(0,0046)$ \\
\hline
\end{tabular}

* Para o CAPM estimado por mínimos quadrados ordinários (painéis A e C), o beta é dado pelo coeficiente do retorno do mercado, estimado sobre a amostra inteira. Para o CAPM com aprendizagem (painéis B e D), o beta é o obtido pela média da série das previsões do beta de um período à frente. O período da amostra é I0.7.1987-2.7.2010.

Erros padrão estão entre parênteses.

Fonte: Elaborada pelos autores.

A Figura I apresenta o comportamento das séries temporais do beta previsto para um período à frente, $b_{t+\mathrm{I} \mid t}^{\text {is }}$, e da previsão de sua média de longo prazo $\beta_{t++\mid t}^{i \text {, }}$, resultantes do filtro de Kalman para os dois modelos da Tabela I. Os gráficos mostram como a previsão do beta corrente (tracejado) é ancorada à previsão do beta de longo prazo (pontilhado), que por sua vez, evolui de forma suave na maioria das ações, cujo comportamento também é verificado nos betas das carteiras de Adrian e Franzoni (2009). Note também que em mais da metade das ações escolhidas (PETR4, ITUB4, PETR3, AMBV4, BRKM5, VALE3, BBAS3) o beta corrente estimado pelo modelo condicional varia muito mais do que o beta corrente estimado pelo modelo incondicional. 


\section{FIGURA I}

BETAS ESTIMADOS PELO FILTRO DE KALMAN

PAINEL A: CAPM INCONDICIONAL COM APRENDIZAGEM
PAINEL B: CAPM CONDICIONAL COM APRENDIZAGEM

\section{PETR4}
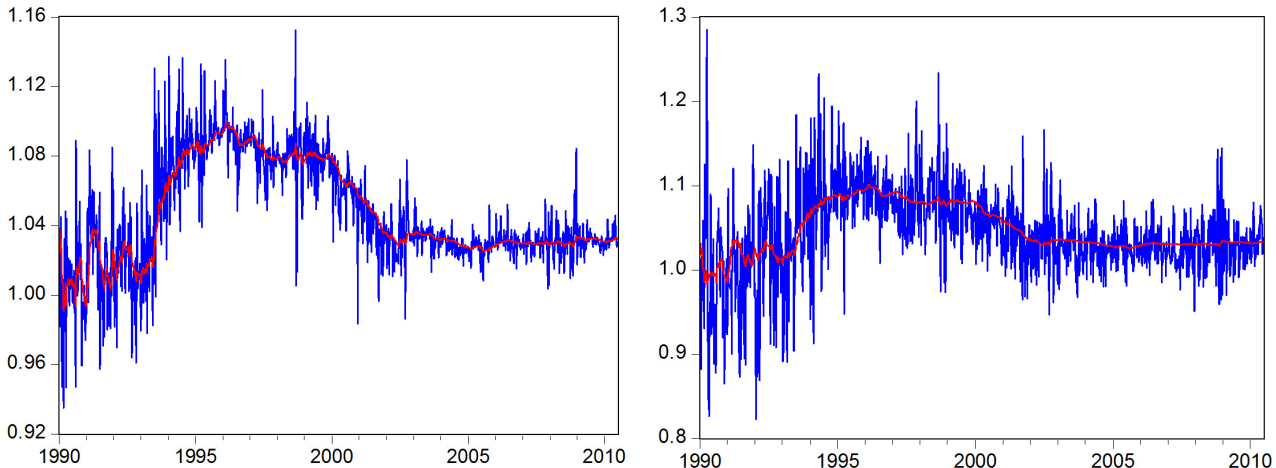

beta $\longrightarrow$ BETA

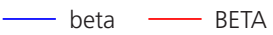

\section{VALE5}
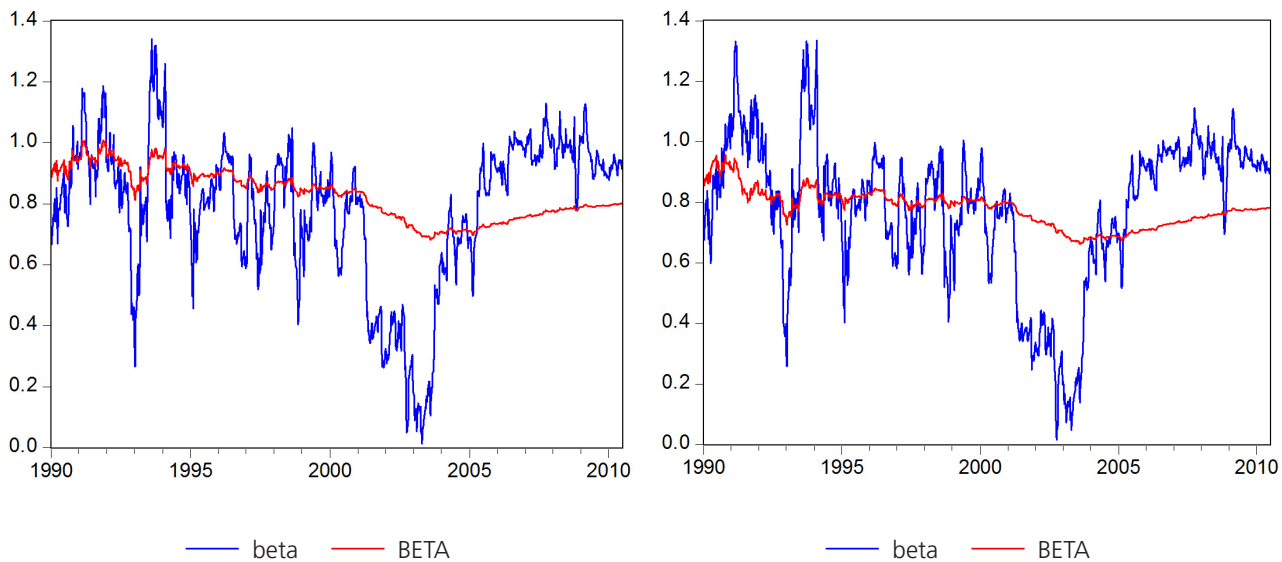

(continua) 


\section{FIGURA I (CONTINUAÇÃO)}

BETAS ESTIMADOS PELO FILTRO DE KALMAN

PAINEL A: CAPM INCONDICIONAL

COM APRENDIZAGEM
PAINEL B: CAPM CONDICIONAL

COM APRENDIZAGEM

\section{BBDC4}
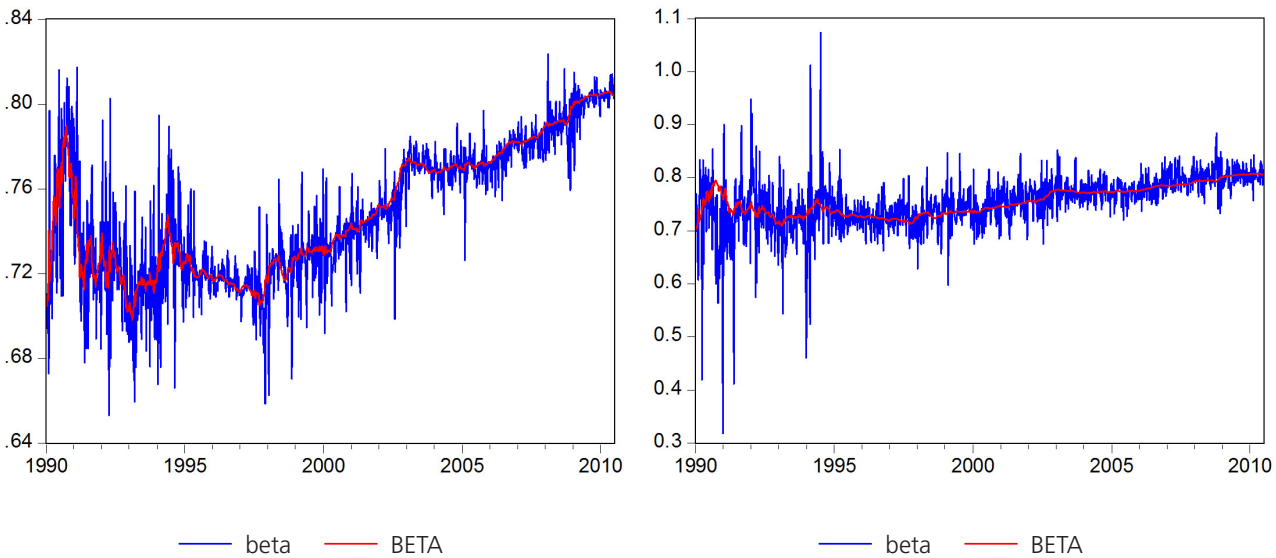

beta $\longrightarrow$ BETA

\section{CEMIG4}
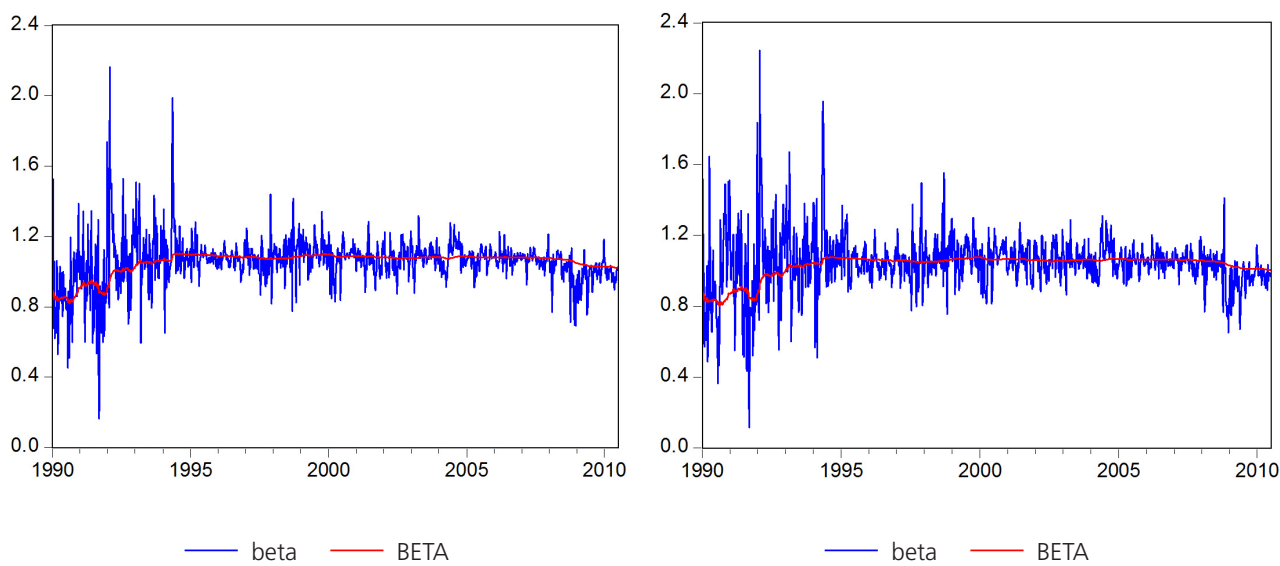


\section{FIGURA I (CONTINUAÇÃO)}

\section{BETAS ESTIMADOS PELO FILTRO DE KALMAN}

PAINEL A: CAPM INCONDICIONAL

COM APRENDIZAGEM
PAINEL B: CAPM CONDICIONAL

COM APRENDIZAGEM

\section{ITUB4}
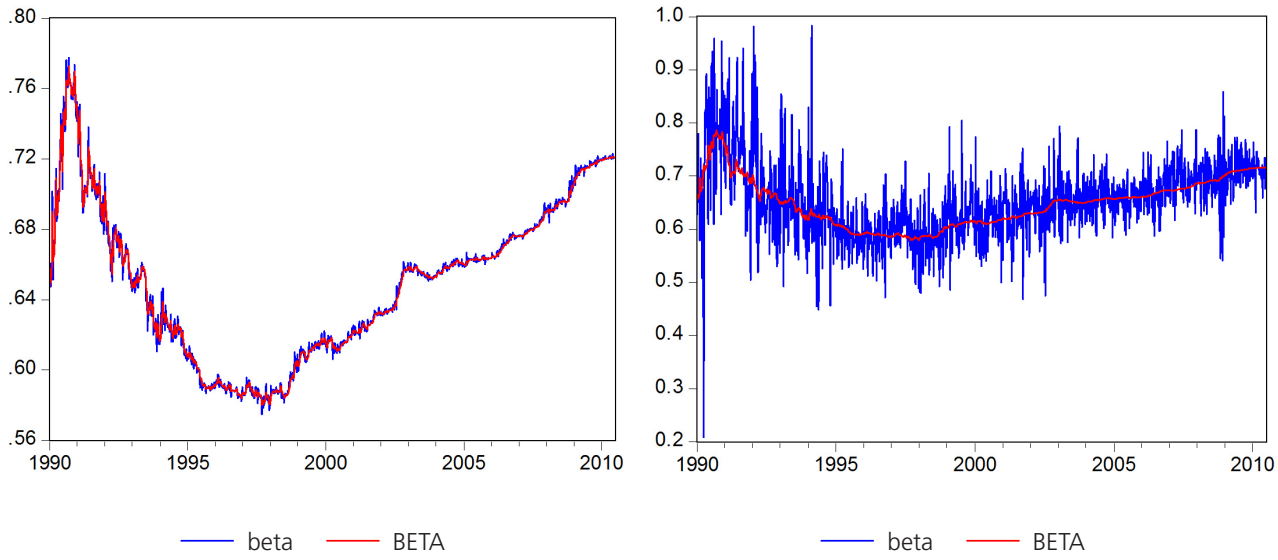

TLPP3
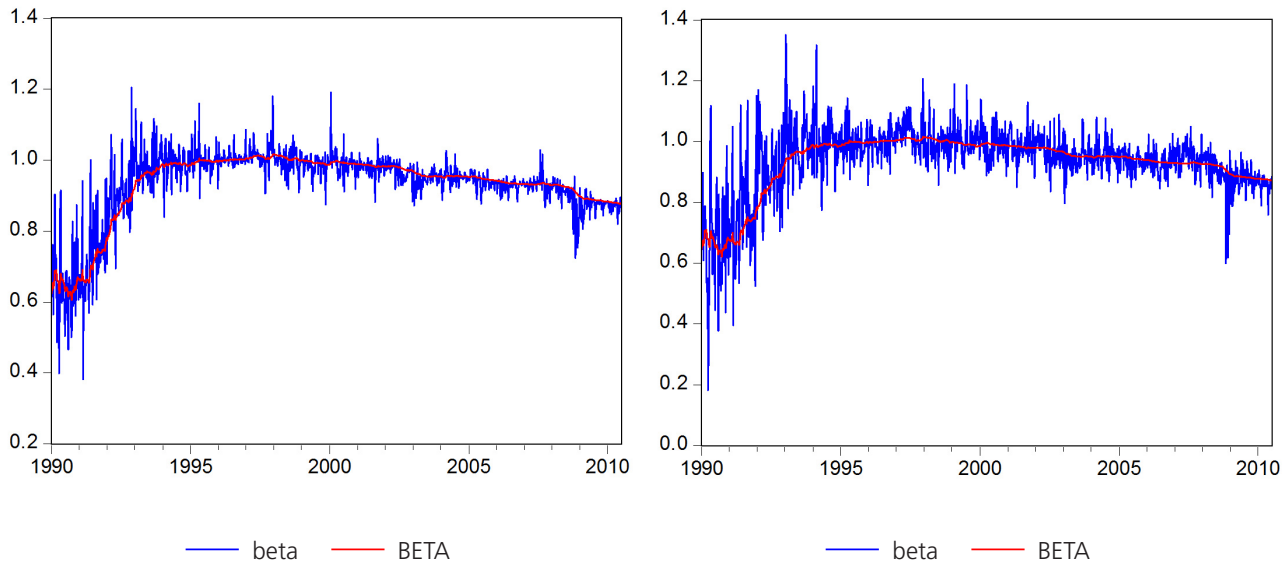

(continua) 


\section{FiguRA I (CONTINUAÇÃO)}

BETAS ESTIMADOS PELO FILTRO DE KALMAN

PAINEL A: CAPM INCONDICIONAL

COM APRENDIZAGEM
PAINEL B: CAPM CONDICIONAL

COM APRENDIZAGEM

\section{PETR3}
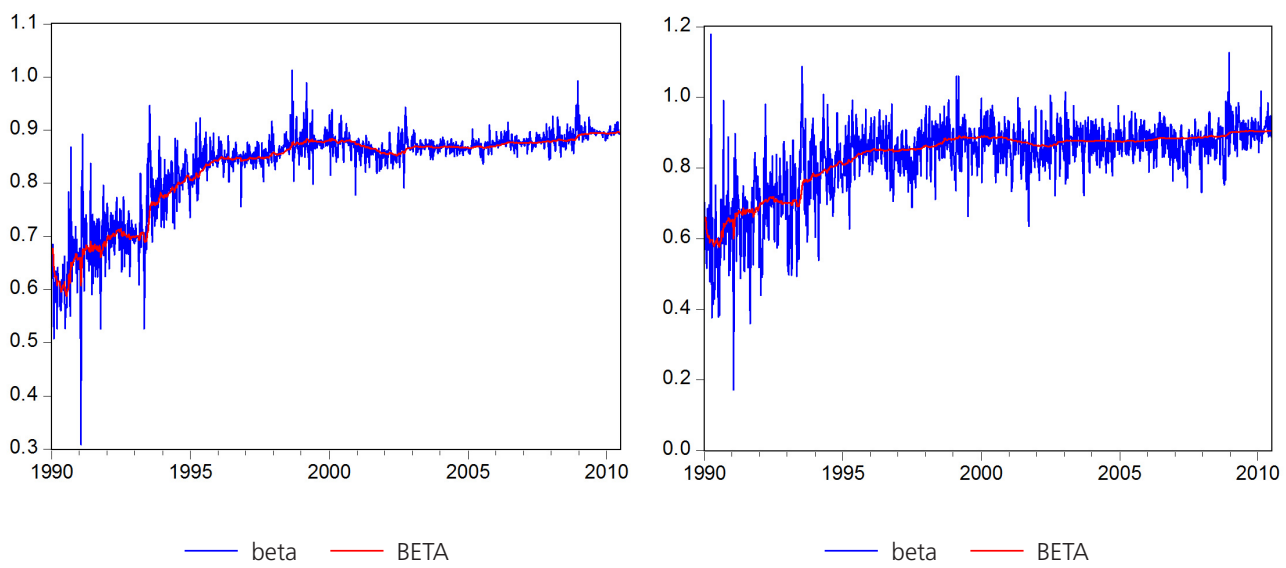

AMBV4
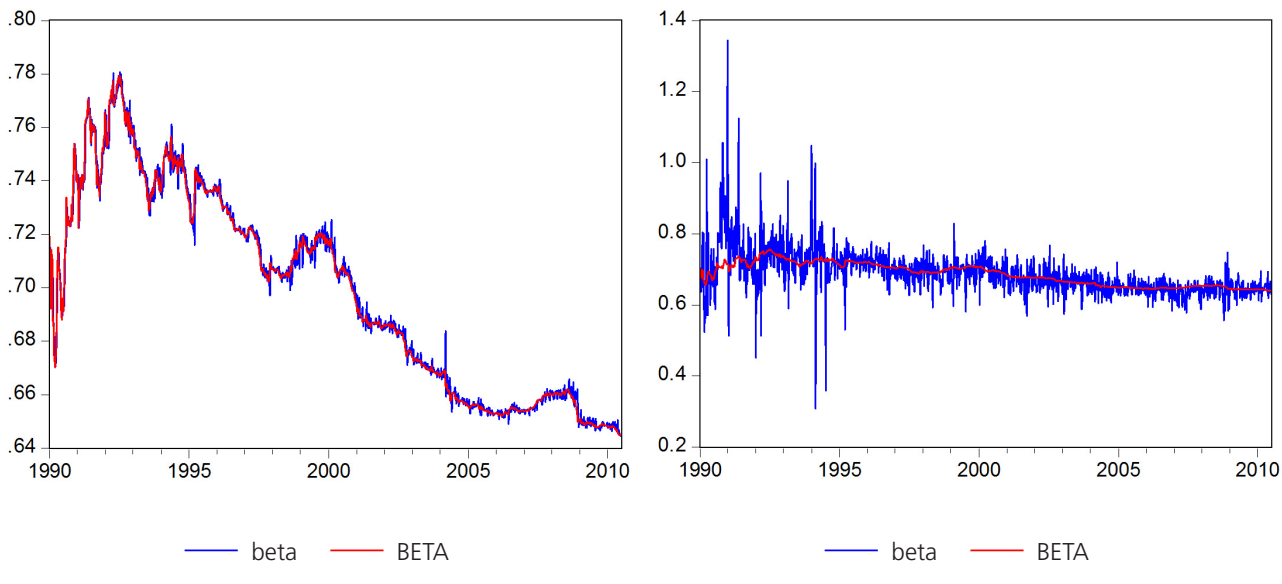


\section{FIGURA I (CONTINUAÇÃO)}

BETAS ESTIMADOS PELO FILTRO DE KALMAN

PAINEL A: CAPM INCONDICIONAL

COM APRENDIZAGEM
PAINEL B: CAPM CONDICIONAL

COM APRENDIZAGEM

ITSA4
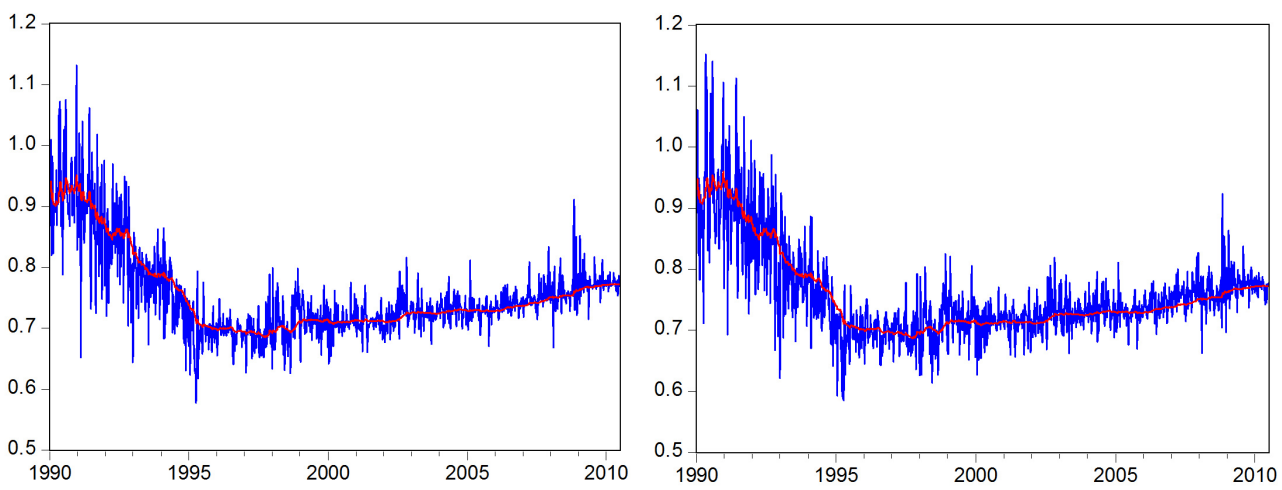

beta - BETA

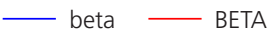

BRKM5
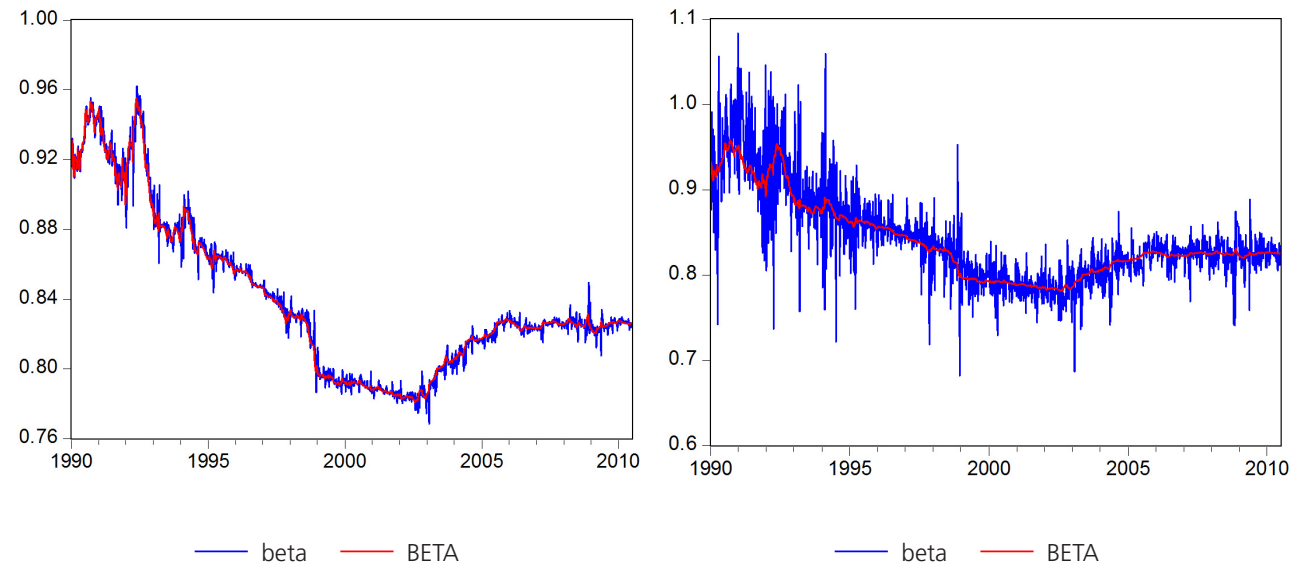

(continua) 


\section{FiguRA I (CONTINUAÇÃO)}

BETAS ESTIMADOS PELO FILTRO DE KALMAN

\begin{tabular}{cc}
\hline $\begin{array}{c}\text { PAINEL A: CAPM INCONDICIONAL } \\
\text { COM APRENDIZAGEM }\end{array}$ & $\begin{array}{c}\text { PAINEL B: CAPM CONDICIONAL } \\
\text { COM APRENDIZAGEM }\end{array}$ \\
\hline & VALE3 \\
\hline
\end{tabular}
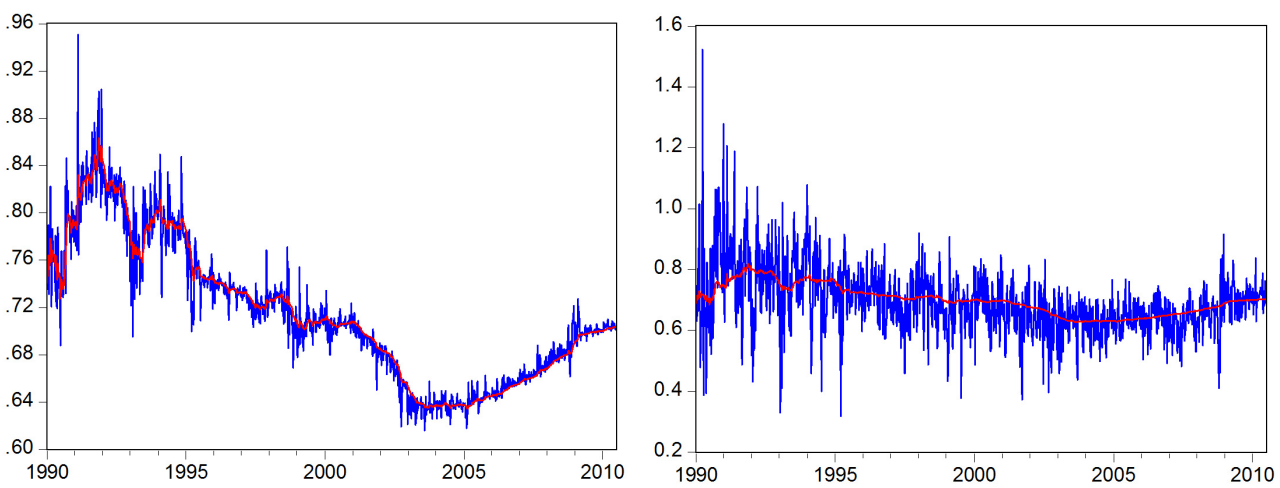

beta - BETA

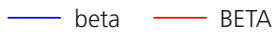

FIBR3



\section{FigURA I (CONCLUSÃO)}

\section{BETAS ESTIMADOS PELO FILTRO DE KALMAN}

PAINEL A: CAPM INCONDICIONAL

COM APRENDIZAGEM
PAINEL B: CAPM CONDICIONAL

COM APRENDIZAGEM

\section{BBAS3}
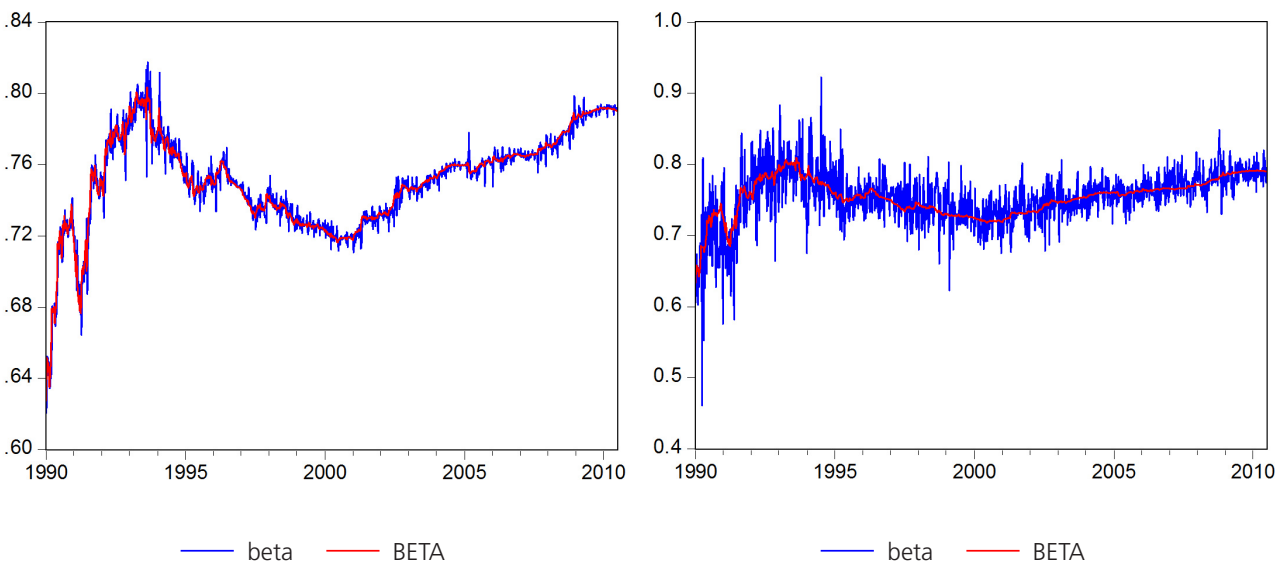

O Painel A corresponde aos betas estimados pelo CAPM incondicional, e o Painel B refere-se aos betas do CAPM com aprendizagem condicionado a MKT defasado, HML e TERM. Em minúsculo, beta representa a série do beta previsto para um período a frente $b_{t+\mathrm{I} \mid t}$, e BETA (em maiúsculo) representa a série da previsão do beta de longo prazo $B_{t+1 \mid t}^{i s}$. Os betas foram estimados sobre a amostra inteira (I0.7.I987 e 2.7.20IO) e representados graficamente a partir 5.I.I990.

Fonte: Elaborada pelos autores.

\section{ERROS DE APREÇAMENTO DO MODELO CAPM COM APRENDIZAGEM}

Adrian e Franzoni (2009) observam que o modelo CAPM com aprendizagem requer um período suficientemente longo para que as expectativas sobre os betas de longo prazo sejam formadas pelos agentes. Neste artigo, apesar de o filtro de Kalman associado ao modelo CAPM com aprendizagem ser inicializado em I0.7.I987 e estimado até 2.7.20I0, os erros de apreçamento são calculados somente para o período pós-Plano Real (6.I.I995-2.7.2010). Dessa forma, de I0.7.I987 a 6.I.I995, as expectativas sobre os betas de longo prazo são formadas e os erros de apreçamento dos modelos são comparados no intervalo de 6.I.I995 até 2.7.2010. 
O erro de apreçamento da ação $i$ com base no modelo CAPM com aprendizagem, $\widehat{\alpha}_{t+1}^{i}$, seja com ou sem variáveis condicionantes, é dado por:

$$
\widehat{\alpha}_{t+\mathrm{I}}^{i}=R_{t+\mathrm{I}}^{i}-\widehat{\beta}_{t+\mathrm{I} \mid t}^{k f} R_{t+\mathrm{I}}^{M}
$$

em que $\widehat{\beta}_{t+\mathrm{IIt}}^{k f}$ é a previsão de um passo à frente do beta feita no período $t$ obtida pelo filtro de Kalman, e $R_{t+1}^{i}$ e $R_{t+1}^{M}$ se referem aos retornos observados da ação $i$ e do mercado, respectivamente. A média de $\widehat{\alpha}_{t+1}^{i}$ do período de 6.I.I995 até 2.7.20I0, denotada por $\bar{\alpha}^{i}$, é utilizada como a estimativa final do erro de apreçamento da ação $i$. Assim, para o cálculo do erro de apreçamento do CAPM com aprendizagem, consideramos as duas especificações apresentadas na Tabela r: a primeira é o modelo estimado sem variáveis condicionantes, denotado por CAPM incondicional com aprendizagem; e a segunda se refere ao CAPM com aprendizagem utilizando as variáveis condicionantes MKT, HML e TERM.

Para o cálculo do erro de apreçamento do modelo CAPM estimado por MQO, consideramos duas especificações: a primeira se refere ao CAPM incondicional; e a segunda, ao CAPM condicional, em que o retorno das ações é regredido em função do retorno de mercado escalonado pelas variáveis condicionantes defasadas MKT, HML e TERM. Essa última especificação é equivalente a um modelo multifator, no qual os fatores adicionais são as interações entre o retorno de mercado e as variáveis condicionantes, ou seja:

$$
\begin{gathered}
R_{t+\mathrm{I}}^{i}=\alpha^{i}+\beta^{i} R_{t+\mathrm{I}}^{M}+\epsilon_{t+\mathrm{I}} \\
R_{t+\mathrm{I}}^{i}=\alpha^{i}+\beta^{\mathrm{Ii}} R_{t+\mathrm{I}}^{M}+\beta^{2 i} R_{t+\mathrm{I}}^{M} R_{t}^{M}+\beta^{3 i} R_{t+\mathrm{I}}^{M} H_{t}+\beta^{4 i} R_{t+\mathrm{I}}^{M} \text { Term }_{t}+\epsilon_{t+\mathrm{I}}
\end{gathered}
$$

em que $\epsilon_{t+1}$ é um choque puramente idiossincrático. Da mesma forma que no CAPM com aprendizagem, as duas especificações do CAPM sem aprendizagem são estimadas sobre toda a amostra (de I0.7.1987 a 2.7.20I0), e seus erros de apreçamento são calculados no período pós-Plano Real (6.I.I995-2.7.2010) por meio das seguintes equações:

$$
\begin{gathered}
\hat{\alpha}^{i}=\bar{R}^{i}-\widehat{\beta}^{i} \bar{R}^{M} \\
\widehat{\alpha}^{i}=\bar{R}^{i}-\widehat{\beta}^{\mathrm{Ii}} \bar{R}^{M}-\widehat{\beta}^{2 i} \bar{\gamma}^{1}-\widehat{\beta}^{3 i} \bar{\gamma}^{2}-\widehat{\beta}^{4 i} \bar{\gamma}^{3}
\end{gathered}
$$

Os coeficientes $\widehat{\beta}^{i}, \widehat{\beta}^{\mathrm{ri}}, \widehat{\beta}^{2 i}, \widehat{\beta}^{3 i}$ e $\widehat{\beta}^{4 i}$ são estimados utilizando toda a amostra (I0.7.I987- 2.7.20I0); e $\bar{R}^{i}, \bar{R}^{M}, \bar{\gamma}^{1}, \bar{\gamma}^{2}$ e $\bar{\gamma}^{3}$ são as médias das séries observadas $R_{t+\mathrm{I}}^{i}$, $R_{t+\mathrm{I}}^{M}, R_{t+\mathrm{I}}^{M} R_{t}^{M}, R_{t+\mathrm{I}}^{M} \mathrm{Hml}_{t}$ e $R_{t+\mathrm{I}}^{M}$ Term $_{t}$, respectivamente, calculadas no intervalo de 6.I.I995 a 2.7.20IO. 
A Tabela 4 apresenta os erros de apreçamento calculados com base nas equações II, I4 e I5. A fim de comparar o desempenho dos diferentes modelos, duas estatísticas de resumo são reportadas nessa tabela. A primeira é a raiz quadrada da média dos erros de apreçamento quadráticos (root mean squared error - RMSE), calculada para cada modelo. A segunda estatística é o erro de apreçamento composto (composite pricing error - CPE) proposto por Campbell e Vuolteenaho (2004), o qual é definido como $C P E=\alpha^{\prime} \Omega^{-1} \alpha$, em que $\alpha$ é um vetor dos erros de apreçamento de cada ação, e $\Omega$ é uma matriz diagonal cujos elementos de sua diagonal principal são as variâncias dos retornos observados de cada ação. Essa medida agregada de erro de apreçamento dá menos peso para os erros de apreçamento das ações mais voláteis, o que possibilita uma comparação mais adequada entre os modelos.

\section{TABELA 4}

ERROS DE APREÇAMENTO: $\bar{\alpha}^{i}$

\begin{tabular}{lcccc}
\hline & PAINEL A & PAINEL B & PAINEL C & PAINEL D \\
\hline AÇÕES & CAPM & $\begin{array}{c}\text { CAPM } \\
\text { INCONDICIONAL }\end{array}$ & $\begin{array}{c}\text { COM APRENDIZAGEM } \\
\text { CAPM CONDICIONAL: }\end{array}$ & $\begin{array}{c}\text { MKT, HML E TERM } \\
\text { COM APRENDIZAGEM: } \\
\text { MKT, HML E TERM }\end{array}$ \\
\hline PETR4 & $0,1252(0,1365)$ & $0,1219(0,1359)$ & $0,1266(0,1365)$ & $0,1305(0,1364)$ \\
\hline VALE5 & $0,1402(0,1608)$ & $0,1586(0,1534)$ & $0,1432(0,1602)$ & $0,1506(0,1531)$ \\
\hline BBDC4 & $0,1188(0,1432)$ & $0,1153(0,1442)$ & $0,1116(0,1426)$ & $0,1188(0,1437)$ \\
\hline CMIG4 & $0,0160(0,1560)$ & $-0,0158(0,1538)$ & $0,0190(0,1565)$ & $0,0045(0,1530)$ \\
\hline ITUB4 & $0,1893(0,1455)$ & $0,1803(0,1489)$ & $0,1973(0,1445)$ & $0,1775(0,1486)$ \\
\hline TLPP3 & $-0,0330(0,1568)$ & $-0,0273(0,1559)$ & $-0,0037(0,1576)$ & $-0,0329(0,1557)$ \\
\hline PETR3 & $0,1811(0,1623)$ & $0,1743(0,1619)$ & $0,1860(0,1628)$ & $0,1721(0,1622)$ \\
\hline AMBV4 & $0,1849(0,1518)$ & $0,1859(0,1510)$ & $0,1721(0,1507)$ & $0,1853(0,1507)$ \\
\hline ITSA4 & $0,1907(0,1381)$ & $0,1735(0,1378)$ & $0,1917(0,1380)$ & $0,1699(0,1377)$ \\
\hline BRKM5 & $-0,1633(0,2014)$ & $-0,1635(0,2017)$ & $-0,1616(0,2015)$ & $-0,1673(0,2019)$ \\
\hline VALE3 & $0,0868(0,1727)$ & $0,0923(0,1731)$ & $0,0904(0,1726)$ & $0,0817(0,1719)$ \\
\hline FIBR3 & $-0,1340(0,2115)$ & $-0,1373(0,2111)$ & $-0,1396(0,2114)$ & $-0,1491(0,2099)$ \\
\hline & & & & \\
\hline
\end{tabular}




\section{TABela 4 (Conclusão)}

ERROS DE APREÇAMENTO: $\bar{\alpha} \%$

\begin{tabular}{lcccc}
\hline & PAINELA & PAINEL B & PAINEL C & PAINEL D \\
\hline AÇÕES & $\begin{array}{c}\text { CAPM } \\
\text { INCONDICIONAL }\end{array}$ & $\begin{array}{c}\text { CAPM } \\
\text { INCONDICIONAL } \\
\text { COM APRENDIZAGEM }\end{array}$ & $\begin{array}{c}\text { CAPM CONDICIONAL: } \\
\text { MKT, HML E TERM }\end{array}$ & $\begin{array}{c}\text { CAPM CONDICIONAL } \\
\text { COM APRENDIZAGEM: } \\
\text { MKT, HML E TERM }\end{array}$ \\
\hline BBAS3 & $-0,0430(0,1733)$ & $-0,0456(0,1737)$ & $-0,0455(0,1727)$ & $-0,0393(0,1732)$ \\
RMSE & 0,136997 & 0,135322 & 0,137306 & 0,135229 \\
\hline CPE & 0,006939 & 0,006781 & 0,006909 & 0,006725 \\
\hline
\end{tabular}

* Os erros de apreçamento são calculados para o período de 6.I.I995 a 2.7.20Io. Painéis A e B apresentam os erros de apreçamento do capm estimado via mqo sem variáveis condicionantes e do CAPM com aprendizagem sem variáveis condicionantes. Nos painéis $\mathrm{C}$ e $\mathrm{D}$ temos os erros de apreçamento do CAPM estimado via ols condicionado às variáveis defasadas mkt, hml e term e do CAPM com aprendizagem condicionado ao retorno de mercado defasado (mkt), ao hml e term.

Erros padrão estão entre parênteses.

Fonte: Elaborada pelos autores.

Iniciamos nossa análise com dois modelos sem variáveis condicionantes a fim de avaliarmos a contribuição da aprendizagem sobre os betas na redução dos erros de apreçamento. Os painéis A e B da Tabela 4 comparam os erros de apreçamento gerados pelo CAPM incondicional (Equação I2) com os do CAPM com aprendizagem. Analisando as duas medidas agregadas, na passagem do CAPM incondicional para o CAPM incondicional com aprendizagem, podemos ver uma redução de I,22\% no RMSE e de 2,29\% no CPE no erro de apreçamento semanal.

Na segunda parte desta análise, avaliamos a contribuição das variáveis condicionantes na redução dos erros de apreçamento. Especificamente, avaliamos se a combinação de variáveis condicionantes com o modelo CAPM com aprendizagem é capaz de reduzir os erros de apreçamento do CAPM, como apontado por Adrian e Franzoni (2009). O Painel C da Tabela 4 apresenta os erros de apreçamento do CAPM condicional estimado por MQO (Equação I3). Ao compararmos os resultados obtidos com o CAPM condicional com os obtidos com CAPM incondicional no Painel A, podemos observar um pequeno aumento de $0,23 \%$ no RMSE e uma pequena redução de $0,44 \%$ no CPE. Apesar do resultado ambíguo, isso sugere que a inclusão de variáveis condicionantes não resulta necessariamente em uma melhoria no desempenho do CAPM estimado via MQO, contrariando as premissas de Jagannathan e Wang (I996) e Lettau e Ludvigson (200I). Tambosi Filho, Garcia e Bertucci (2007) utilizam as metodologias de 
Fama e MacBeth (I974) e de Jagannathan e Wang (I996) e testam o CAPM condicional no Brasil, na Argentina e nos Estados Unidos para o período de janeiro de 1994 até dezembro de 2002. Eles chegam à conclusão de que o CAPM condicional de Jagannathan e Wang (I996) para o mercado norte-americano é perfeitamente aplicável aos mercados brasileiro e argentino. Em relação ao modelo condicional, o poder de explicação do modelo aumenta muito para cada país analisado. Apesar de essas evidências empíricas favorecerem a não rejeição do CAPM condicional nesses países, Lewellen e Nagel (2006) argumentam que a estrutura correta para testar o CAPM condicional é utilizar séries temporais e não cross-section, como tem sido utilizado por Jagannathan e Wang (I996), Lettau e Ludvigson (200I) e Tambosi Filho, Garcia e Bertucci (2007). Lewellen e Nagel (2006) testam o CAPM condicional no mercado americano utilizando 25 carteiras similares às utilizadas por Adrian e Franzoni (2009) e analisam o modelo utilizando dados diários, semanais e mensais dos retornos durante o período de julho de I964 até junho de 200 I. Eles utilizam várias variáveis condicionantes, incluindo a variável CAY de Lettau e Ludvigson (200I) para testar o modelo. No entanto, chegam à conclusão de que o CAPM condicional produz estimações tão fracas quanto o CAPM incondicional. Lewellen e Nagel (2006) argumentam ainda que os valores dos alfas condicionais são altos e significantes estatisticamente, o que representa uma violação direta do CAPM condicional. Por fim, embora o CAPM condicional reduza o erro de apreçamento relativo ao CAPM incondicional no trabalho de Adrian e Franzoni (2009), a melhoria não é substancial, consistente também com as predições de Lewellen e Nagel (2006).

O Painel D da Tabela 4 apresenta os erros de apreçamento do CAPM condicional com aprendizagem, utilizando o retorno de mercado defasado $M K T$, e as variáveis $H M L$ e TERM como instrumentos de informação para o beta condicional. Note que, quando se comparam os erros de apreçamento desse modelo com os do CAPM incondicional (Painel A), as medidas RMSE e CPE caem I,29\% e 3,09\%, respectivamente. Quando se efetua a mesma comparação com o CAPM condicional (Painel C), as duas medidas agregadas sofrem reduções similares, de I,51\% no RMSE e de $2,66 \%$ no CPE. Por fim, ao compararmos o CAPM condicional com aprendizagem com o CAPM incondicional com aprendizagem, podemos verificar reduções muito pequenas de $0,07 \%$ no RMSE e de $0,83 \%$ no CPE. Apesar de pequenas, essas reduções sugerem que a inclusão de variáveis condicionantes no CAPM com aprendizagem pode contribuir para a redução de seus erros de apreçamento, o que leva à conclusão de que, se especificado corretamente em regressões temporais, o CAPM condicional é capaz de superar as outras versões. Com isso, o CAPM condicional com aprendizagem se apresentou como o melhor modelo, com os menores valores de RMSE e CPE dentro de todos os modelos analisados. 
Os resultados obtidos neste artigo corroboram a evidência empírica anterior reportada por Adrian e Franzoni (2009), pois sugerem que a inclusão de variáveis condicionantes no CAPM com aprendizagem é capaz de provocar reduções nos erros de apreçamento quando comparado ao modelo CAPM em sua versão original.

Vale ressaltar, entretanto, que as reduções nos erros de apreçamento reportadas neste artigo foram inferiores àquelas encontradas por Adrian e Franzoni (2009). Essa diferença nos resultados se deve a uma série de possíveis razões. Primeiro, os dados utilizados foram coletados com frequências diferentes (trimestral em Adrian e Franzoni e semanal neste artigo). Segundo, Adrian e Franzoni (2009) utilizam carteiras na sua análise e não ativos individuais. Sobre esse aspecto, Lo (2004) argumenta que o agrupamento de ações com base em diferentes atributos pode resultar em diferentes inferências, mesmo utilizando o mesmo conjunto de ações. Em conjunto, esses fatores podem contribuir para que o ajuste do modelo de espaço de estados associado ao CAPM com aprendizagem seja melhor. Além disso, é importante ressaltar que nosso estudo esteve limitado ao pequeno número de ações, pois a escolha das ações deu-se levando em conta o critério de liquidez e utilizando apenas ações que permaneceram cotadas na Bovespa durante todo o período analisado, no entanto, devido ao pequeno grau de liquidez no mercado brasileiro, foram escolhidas poucas ações sobreviventes. E a escolha por dados semanais se deve ao fato de que utilização de dados mensais ou trimestrais, tal como feito em Adrian e Franzoni (2009), reduziria o tamanho da amostra, pois os dados de ações no Brasil estão disponíveis somente a partir de I986. Essas limitações não são encontradas no mercado americano, o qual dispõe de uma longa base de dados e apresenta um grau de liquidez muito maior do que no mercado brasileiro, permitindo estimações mais robustas. Dessa forma, tal limitação no que toca o nível de liquidez e a disponibilidade de ações cotadas ao longo do período estudado podem ter afetado as estimações deste trabalho. De qualquer forma, vale enfatizar que os resultados encontrados neste artigo corroboram a evidência empírica anterior baseada no mercado acionário norte-americano. Em resumo, concluímos que, na medida em que a aprendizagem sobre o risco sistemático é introduzida, o modelo CAPM é capaz de apresentar um melhor desempenho em termos de erros de apreçamento.

\section{CONCLUSÃO}

Este trabalho aplicou o modelo CAPM condicional com aprendizagem proposto por Adrian e Franzoni (2009) em I3 ações com alto índice de liquidez do 
mercado brasileiro, durante o período de I0.7.1987 a 2.7.20Io. Foi avaliado se esse modelo é capaz de melhorar o desempenho do CAPM em termos de erros de apreçamento. Os resultados empíricos indicaram que a introdução da aprendizagem sobre o beta na estimação do CAPM é capaz de reduzir os erros de apreçamento tanto no CAPM incondicional quanto no CAPM condicional. Além disso, a inclusão de variáveis condicionantes na estimação do beta levou a reduções no erro de apreçamento ainda maiores. Nesse sentido, os resultados deste artigo corroboram a evidência empírica anterior reportada por Adrian e Franzoni (2009) e baseada no mercado acionário norte-americano.

Dessa forma, convém ressaltar que testes empíricos da natureza dos pretendidos nesta pesquisa ainda não foram realizados com o modelo CAPM aplicado aos países da América da Sul, e muito menos utilizando o CAPM condicional.

Com isso, sugere-se esse mesmo modelo em outros países da América do Sul, tal como feito por Tambosi Filho, Garcia e Bertucci (2007), a fim de verificar se, ao levar em conta a aprendizagem, o CAPM condicional é capaz de produzir erros menores não somente no mercado norte-americano.

Como temas futuros de pesquisa, sugere-se primeiramente a análise de outras variáveis exógenas na equação do beta condicional, a fim de avaliar seu impacto no erro de apreçamento derivado do modelo CAPM com aprendizagem. Além disso, é interessante avaliar a robustez dos resultados utilizando carteiras em vez de ativos individuais, com o objetivo de avaliar se esse procedimento pode reduzir os erros de apreçamento e explicar uma fração do efeito valor. Isso também permitiria explorar resultados mais teóricos envolvendo as anomalias do efeito valor e de tamanho encontradas nos estudos do CAPM, tal como feito em Adrian e Franzoni (2009). Finalmente, algumas hipóteses feitas neste artigo poderiam ser relaxadas, como a suposição de que os betas seguem um processo linear autorregressivo com inovações homocedásticas ou até mesmo estimar o parâmetro alfa em uma equação de estado, permitindo variar no tempo como o beta, tal como feito em Trecroci (2009). Implementaremos essas sugestões em trabalhos futuros.

\section{CONDITIONAL CAPM WITH LEARNING APPLIED TO THE BRAZILIAN STOCK MARKET}

\section{ABSTRACT}

Asset pricing models represent one of the most discussed and researched areas in finance. They are widely used in a theoretical and practical manner to model 
and predict risk and return to price securities and portfolios as well as in corporate finance to estimate the cost of capital and rank investment projects. They provide a usable measure of risk that helps managers and investors determine what return they deserve for putting their money at risk. The objective of this paper is to analyze the performance of the learning-augmented conditional CAPM model of Adrian and Franzoni (2009) when applied to the returns of the most liquid stocks transactioned in the Brazilian stock market from I987 to 20I0. Adrian and Franzoni (2009), in their paper, complemented the conditional CAPM literature by modeling a new type of time-variation in conditional betas. In this environment, investors form expectations about the long run level of factor loadings from the observation of realized returns of exogenous variables. As a direct consequence of this assumption, conditional betas are modeled using the Kalman filter. Using data of 25 portfolios sorted by size and book-to-market ratio, the authors concluded that the learning-augmented conditional CAPM is able to substantially reduce the pricing errors when compared to the original version of CAPM. Thus, we contribute to the pricing asset literature, as we evaluate whether this model is able to reduce pricing errors in relation to its original version when applied to Brazilian individual asset data. The results of this article showed a decreasing in the pricing errors of learning-augmented conditional CAPM in relation to CAPM in its original version. Our empirical results suggests that the learning about betas should be taken into account when estimating both conditional and unconditional CAPM.

\section{KEYWORDS}

Conditional CAPM; Kalman filter; Forecasting; Beta coefficient; Pricing errors.

\section{CAPM CONDICIONAL CON EL APRENDIZAJE APLICADO A LA BOLSA DE VALORES BRASILEÑA}

\section{RESUMEN}

Modelos de valoración de activos representan una de las más discutidas e investigadas áreas en las finanzas. Son ampliamente utilizados de forma teórica y práctica en el área de investimentos para modelizar y predecir el riesgo y la rentabilidad de los títulos y de las carteras, así como en finanzas corporativas para estimar el coste de capital y clasificar proyectos de inversión. Proporcionan una medida útil de riesgo a los gestores y a los inversores para ayudarles a deter- 
minar el retorno adecuado para poner su dinero en riesgo. El objetivo de este trabajo es analizar el desempeño del modelo CAPM condicional con aprendizaje propuesto por Adrian y Franzoni (2009) cuando aplicado a los retornos de las acciones más negociadas en el mercado de valores de Brasil desde 1987 hasta 20I0. Adrian y Franzoni (2009), en su artículo, contribuyen a la literatura del CAPM condicional por proponer un nuevo tipo de variación temporal en los betas condicionales. En este entorno, los inversores se forman expectativas sobre el nivel de largo plazo de los pesos factoriales a partir de la observación de los rendimientos realizados de variables exógenas. Como consecuencia directa de este supuesto, los betas condicionales se pueden modelizar mediante el filtro de Kalman. Utilizando datos de 25 acciones ordenadas por el tamaño y por el índice de valor contable-valor de mercado, los autores concluyeron que el CAPM condicional con el aprendizaje es capaz de reducir sustancialmente los errores de precios en comparación con el CAPM en su versión original. De esta manera, contribuimos con la literatura de valoración de activos, al evaluar si este modelo es capaz de reducir los errores de precios en relación a la versión original del modelo CAPM, cuando aplicada a los datos del mercado brasileño a partir de los activos individuales. Los resultados de este estudio muestran una reducción de lo modelo CAPM condicional con aprendizaje en relación con el CAPM en su versión original. Por lo tanto, os resultados empíricos sugieren que el aprendizaje acerca de los betas debe ser tomado en cuenta en la estimación del CAPM condicional e incondicional.

\section{PALABRAS CLAVE}

CAPM condicional; Filtros de Kalman; Previsión; Coeficiente beta; Errores de valoración.

\section{REFERÊNCIAS}

ADRIAN, T.; FRANZONI, F. Learning about beta: a new look at CAPM tests. Staff Reports of Federal Reserve Bank of New York, n. I93, p. I-47, 2004.

ADRIAN, T.; FRANZONI, F. Learning about beta: time-varying factor loadings, expected returns, and the conditional CAPM. Journal of Empirical Finance, v. I6, n. 4, p. 537-556, 2009.

CAMPBELL, J.; VUOLTEENAHO, T. Bad beta, good beta. American Economic Review, v. 94, p. I249-I275, 2004 .

FAMA, E. F.; FRENCH, K. R. The cross-section of expected stock returns. Journal of Finance, v. 47, n. 2, p. 427-465, I992. 
FAMA, E. F.; FRENCH, K. R. Common risk factors in the returns on stocks and bonds. Journal of Financial Economics, v. 33, p. 3-56, I993.

FAMA, E. F.; MAcBETH, J. D. Risk, return and equilibrium: empirical tests. Journal of Political Economy, v. 8I, n. 3, p. 607-636, I973.

HAMILTON, J. D. Time series analysis. Princeton: Princeton University Press, I994.

HUANG, P.; HUENG, C. J. Conditional risk-return relationship in a time-varying beta model. Journal of Quantitative Finance, v. 8, n. 4, p. 38I-390, 2008.

JAGANNATHAN, R.; WANG, Z. The conditional CAPM and the cross-section of expected returns. Journal of Finance, v. 5I, p. 3-53, I996.

KOOPMAN, S. J.; DURBIN, J. Time series analysis by state space methods. Oxford: Oxford University Press, 200I.

LETTAU, M.; LUDVIGSON, S. Resurrecting the (C)CAPM: a cross-sectional test when risk premia are time-varying. Journal of Political Economy, v. I09, p. I238-1287, 2001.

LEWELLEN, J.; NAGEL, S. The conditional CAPM does not explain asset-pricing anamolies. Journal of Financial Economics, v. 82, n. 2, p. 289-314, 2006.

LINTNER, J. The valuation of risk assets and selection of risky investments in stocks portfolios and capital budgets. Review of Economics and Statistics, v. 47, p. 13-37, 1965.

LO, I. Portfolio formation can affect asset pricing tests. Journal of Asset Management, v. 5, n. 3, p. 203-216, 2004 .

MACHADO, A.; MEDEIROS, O. Modelos de precificação de ativos e o efeito liquidez: evidências empíricas no mercado acionário brasileiro. Revista Brasileira de Finanças, v. 9, n. 3, p. 338-4I2, 20 II. MCCULLOCH, J. H. The Kalman foundations of adaptive least squares, with application to U.S. inflation. Ohio: Ohio State University, 2005. Working paper. p. I-36.

MOSSIN, J. Equilibrium in a capital asset market. Econometrica, v. 34, n. 4, p. 768-783, I966.

RAMOS, P.; PICANÇO, M.; COSTA JR., N. C. A. da. Retornos e riscos das value e growth stocks no mercado brasileiro. In: COSTA JR., N. C. A. DA; LEAL, R. P. C.; LEMGRUBER, E. F. (Org.). Mercado de capitais. São Paulo: Atlas, 2000. p. I24-I37.

SHARPE, W. F. Capital asset prices: a theory of market equilibrium under conditions at risk. Journal of Finance, v. I9, p. 425-442, I964.

SILVA FILHO, O. C.; FRASCAROLI, B. F. A evolução dos betas do CAPM - o caso do setor de telecomunicações brasileiro. In: SEMINÁRIOS EM ADMINISTRAÇÃO - SEMEAD, 8. 2005, São Paulo. Anais... São Paulo: FEA-USP, 2005.

TAMBOSI FILHO, E.; GARCIA, F. G. Modelo CAPM condicional: um panorama geral. Revista de Economia Mackenzie, v. 5, n. 5, p. I29-I40, 2007.

TAMBOSI FILHO, E.; GARCIA, F. G.; BERTUCCI, L. A. Testando empiricamente o CAPM condicional dos retornos esperados de carteiras dos mercados brasileiro, argentino e norte-americano. Revista de Gestão USP, v. I4, n. 4, p. 63-75, 2007.

TRECROCI, C. How do alphas and betas move? Uncertainty, learning and time variation in risk loadings. 2009. Disponível em: <http://ssrn.com/abstract=I498752>. Acesso em: I2 mar. 201 I. 


\section{APÊNDICE A}

\section{CONSTRUÇÃO DO SPREAD DE VALOR HML PARA OS DADOS BRA- SILEIROS SEMANAIS}

Para a formação das carteiras de valor e de crescimento, a população analisada foi composta de todas as empresas com ações listadas na Bolsa de Valores de São Paulo (Bovespa) entre I0.7.I987 e 2.7.20Io, cujos dados foram obtidos no Economática ${ }^{\circledR}$. Foram excluídas da população as empresas:

- do setor financeiro, pois, segundo Fama e French (1992), seu alto grau de endividamento influencia o índice valor contábil-valor de mercado (book-to-market);

- que não apresentaram cotações mensais consecutivas por 24 meses, sendo I2 meses anteriores à data de formação das carteiras e I2 meses posteriores - o objetivo de tal método de seleção se deve ao fato de que a liquidez é um fator importante quando se testam modelos de apreçamento (MACHADO; MEDEIROS, 2OII);

- $\quad$ que não apresentaram P/VPA (razão do preço da ação sobre seu valor patrimonial) positivo em 3I de dezembro do ano anterior à formação das carteiras, com tolerância de 20 dias.

Para as empresas que apresentaram mais de uma ação, foi escolhida a ação que apresentou maior índice de liquidez médio do ano anterior à formação das carteiras.

As carteiras foram montadas em junho de cada ano, para evitar o viés look-ahead bias ${ }^{8}$, começando em junho de 1987 , devido à falta de dados antes de I987. Em seguida, ainda em junho de cada ano, a amostra das ações mais negociadas foi dividida em duas carteiras, a primeira contendo $30 \%$ das ações de menor P/VPA (ações de valor) e a segunda contendo 30\% das ações de maior P/VPA (ações de crescimento). O P/VPA foi utilizado como proxy para o índice book-to-market devido à disponibilidade de seus dados. Além do fato de o P/VPA ser o inverso do índice book-to-market, sendo utilizado também por Ramos et al. (2000) para a formação das suas carteiras de ações de valor e de crescimento.

"Uso de informação ou dados em um estudo ou simulação que não é conhecido ou disponível durante o período que está sendo analisado. Esse viés ocorre quando há a utilização de alguma informação posterior à data de seleção da amostra ou período de simulação, gerando resultados questionáveis. Assim, uma forma de evitar esse viés é garantir aos investidores o conhecimento de informações contábeis relevantes que possam influenciar o preço das ações e, consequentemente, o prêmio de risco das carteiras" (MACHADO; MEDEIROS, no prelo). 
Os preços de fechamento das ações integrantes das duas carteiras foram coletados semanalmente. Por fim, o retorno semanal de cada carteira foi obtido pela média simples dos retornos semanais das ações integrantes. Para a obtenção dos prêmios de risco de cada carteira, foi utilizada a taxa de juros Selic semanal como proxy para o ativo livre de risco.

\section{APÊNDICE B}

\section{DERIVAÇÃO DO FILTRO DE KALMAN ASSOCIADO AO MODELO CAPM COM APRENDIZAGEM}

Seguindo Adrian e Franzoni (2009), o sistema das equações (6) a (8) pode ser representado como:

$$
\begin{gathered}
\xi_{t+\mathrm{I}}^{i}=\tilde{F} \xi_{t}^{i}+\Phi^{i^{\prime}} Y_{t}+U_{t+\mathrm{I}}^{i} \forall i \\
R_{t}^{i}=H_{t}^{\prime} \xi_{t}^{i}+\eta_{t}^{i} \forall i
\end{gathered}
$$

em que:

$$
\xi_{t}^{i}=\left(\begin{array}{c}
B^{i} \\
\beta_{t}^{i}
\end{array}\right) \quad \tilde{F}^{i}=\left(\begin{array}{cc}
\mathrm{I} & 0 \\
\mathrm{I}-F^{i} & F^{i}
\end{array}\right) \quad H_{t}=\left(\begin{array}{c}
0 \\
R_{t}^{M}
\end{array}\right) \quad U_{t+\mathrm{I}}^{i}=\left(\begin{array}{c}
0 \\
u_{t+\mathrm{I}}^{i}
\end{array}\right) \quad \Phi_{t}^{i^{\prime}}=\left(\begin{array}{c}
0 \\
\varnothing^{i^{\prime}}
\end{array}\right) .
$$

Além disso, a matriz de variância-covariância do erro previsto é denotada como:

$$
\Gamma_{t+1 \mid t}^{i}=E_{t}\left[\left(\xi_{t+1}^{i}-E_{t}\left[\xi_{t+1}^{i}\right]\right)\left(\xi_{t+1}^{i}-E_{t}\left[\xi_{t+1}^{i}\right]\right)^{\prime}\right]
$$

Com essa notação, o filtro de Kalman de Hamilton (I994) pode ser diretamente aplicado:

$$
\begin{gathered}
E_{t}\left[\xi_{t+\mathrm{I}}^{i}\right]=\tilde{F}^{i} E_{t}\left[\xi_{t}^{i}\right]+k_{t}^{i}\left(R_{t}^{i}-H_{t}^{\prime} E_{t}\left[\xi_{t}^{i}\right]\right) \\
k_{t}^{i}=\tilde{F}^{i} \Gamma_{t \mid t-\mathrm{I}}^{i} H_{t}\left(H_{t}^{\prime} \Gamma_{t \mid t-\mathrm{I}}^{i} H_{t}+\sigma_{\eta}^{i_{2}}\right)^{-I} .
\end{gathered}
$$


Utilizando a notação introduzida na Seção 2, obtemos:

$$
\left[\begin{array}{c}
B_{t}^{i s} \\
\beta_{t+\mathrm{I} \mid t}^{i s}
\end{array}\right]=\left[\begin{array}{c}
E_{t}\left[B_{t}^{i}\right] \\
E_{t}\left[\beta_{t+\mathrm{I}}^{i}\right]
\end{array}\right]=\left[\begin{array}{cc}
\mathrm{I} & 0 \\
\mathrm{I}-F^{i} & F^{i}
\end{array}\right]\left[\begin{array}{c}
B_{t-\mathrm{I}}^{i s} \\
\beta_{t \mid t-\mathrm{I}}^{i s}
\end{array}\right]+\left[\begin{array}{c}
0 \\
\varnothing^{i^{\prime}} Y_{t}
\end{array}\right]+k_{t}^{i}\left(R_{t}^{i}-E_{t-\mathrm{I}}\left[R_{t}^{i}\right]\right),
$$

em que:

$$
k_{t}^{i}=\left[\begin{array}{cc}
\mathrm{I} & 0 \\
\mathrm{I}-F^{i} & F^{i}
\end{array}\right] \Gamma_{t \mid t-\mathrm{I}}^{i}\left[\begin{array}{c}
0 \\
R_{t}^{M}
\end{array}\right]\left(\left[\begin{array}{c}
0 \\
R_{t}^{M}
\end{array}\right]^{\prime} \Gamma_{t \mid t-\mathrm{I}}^{i}\left[\begin{array}{c}
0 \\
R_{t}^{M}
\end{array}\right]+\sigma_{\eta}^{i_{2}}\right)^{-\mathrm{I}} .
$$

Escrevendo cada equação de atualização separadamente, obtemos as equações 4 e 5, em que:

$$
K_{t}^{i}=\frac{\gamma_{t \mid t-1}^{i(\mathrm{I}, 2)}}{\gamma_{t \mid t-\mathrm{I}}^{i(2,2)}\left(R_{t}^{M}\right)^{2}+\sigma_{\eta}^{i 2}} R_{t}^{M} \quad k_{t}^{i}=\frac{\left(\mathrm{I}-F^{i}\right) \gamma_{t \mid t-\mathrm{I}}^{i(\mathrm{I}, 2)}+\gamma_{t \mid t-\mathrm{I}}^{i(2,2)} F^{i}}{\gamma_{t \mid t-\mathrm{I}}^{i(2,2)}\left(R_{t}^{M}\right)^{2}+\sigma_{\eta}^{i 2}} R_{t}^{M} \quad k_{t}^{i}=\left(K_{t}^{i} k_{t}^{i}\right)^{\prime},
$$

e $\gamma_{t \mid t-\mathrm{I}}^{i(q, r)}$ são os elementos (q-ésimo, r-ésimo) da matriz $\Gamma_{t \mid t-\mathrm{I}}^{i}$, a qual se desenvolve como:

$$
\Gamma_{t \mid t-\mathrm{I}}^{i}=\left(\tilde{F}^{i}-k_{t}^{i} H_{t}^{i^{\prime}}\right) \Gamma_{t \mid t-\mathrm{I}}^{i}\left(\tilde{F}^{i^{\prime}}-H_{t}^{i} k_{t}^{i^{\prime}}\right)+\sigma_{\eta}^{2} k_{t}^{i} k_{t}^{i^{\prime}}+\left(\begin{array}{cc}
0 & 0 \\
0 & \sigma_{u}^{2}
\end{array}\right) .
$$

